\title{
Anomalous States of Positronium
}

\author{
Chris W Patterson
}

Centre for Quantum Dynamics, Griffith University, Nathan QLD 4111 Australid*

\begin{abstract}
The energies and wavefunctions of the two-body Dirac equation for positronium are compared with those of the Pauli approximation and the Bethe-Salpeter equation. The unusual behavior of the ground-state wavefunction of the Dirac equation is explained in terms of anomalous bound-state solutions.
\end{abstract}

${ }^{*}$ Guest Scientist, Theoretical Division, Los Alamos National Laboratory, NM 87545 USA 


\section{INTRODUCTION}

The two-body Dirac equation for positronium, with only a Coulomb potential, has been solved numerically by Scott, Schertzer, and Moore [1] using very accurate finite element methods. In this paper, their Dirac energies and wave functions are compared with the results of the Pauli approximation [2] and also with the solutions to the Bethe-Salpeter equation [3]. The two-body Dirac energies agree with those of the Pauli approximation to a surprisingly high degree of accuracy. On the other hand, their ground-state wave functions differ significantly near the origin. One purpose of this paper is to determine which groundstate wave function is correct. In order to do so one must consider the Bethe-Salpeter equation in detail.

In this paper it is shown that both the Dirac equation and the Bethe-Salpeter equation have two sets of bound-state solutions: the normal 'atomic' solutions and the 'anomalous' solutions in which either the electron or the positron is in a negative-energy state. However, the two-body Dirac equation is not relativistically invariant and does not properly treat the relative time between the electron and positron. As a result of this wrong temporal behavior, the atomic and anomalous states are not orthogonal and can be coupled by the Coulomb interaction. On the other hand, for the relativistically invariant Bethe-Salpeter equation, the atomic and anomalous states are orthogonal because of their different time propagation and there can be no Coulomb coupling between them. Without this coupling to the anomalous states, it is shown that there is no unusual behavior near the origin for the Bethe-Salpeter ground-state wave function. The results in this paper rely heavily on the previous work of Scott, Schertzer, and Moore from Ref. [1].

In Sec. II a simple derivation for the two-body Dirac equation is given in both the coordinate and the momentum representation. This simplification uses recoupling coefficients so that the two-body operators can be readily evaluated using one-body equations. The results in the coordinate representation are shown in Appendix A and agree with previous works [1], [4]. The results in the momentum representation are shown in Appendix B and have not been found in the literature. The momentum representation is useful when calculating the energies and wave functions of both the Pauli states and the anomalous states.

In Sec. III the Dirac energies for positronium in [1] are compared with the Pauli energies and shown to differ by order $m c^{2} \alpha^{6}$ or less. However, the Dirac equation also gives anoma- 
lous bound-state solutions for which the radial wave functions are Dirac delta functions. Unlike the atomic solutions, these anomalous solutions can be obtained most readily in the momentum representation by using the completeness relation for the radial wave functions. It is shown that the unusual ground-state atomic wave function of the Dirac equation is explained by the Coulomb interaction between the atomic and anomalous states. Moreover, in this section, the effect of the magnetic potential on the atomic and anomalous states is derived. This enables one to compare the Dirac energies for the Coulomb potential alone in Ref. [1] with the actual positronium fine structure to order $m c^{2} \alpha^{4}$.

In Sec. IV the Bethe-Salpeter equation for positronium is used to show that the correct atomic state energies and wave functions are obtained by eliminating or 'projecting out' the anomalous states. Indeed, it is shown that the correct atomic bound-states occur only when using the Feynman time propagator $K_{F}$ and the correct anomalous bound-states occur only when using the retarded time propagator $K_{R}$.

\section{TWO-BODY DIRAC EQUATIONS}

The Dirac equation for a free particle is

$$
\begin{aligned}
\boldsymbol{h}_{0} \psi & =\left(c \boldsymbol{\alpha} \cdot \boldsymbol{p}+m c^{2} \beta\right) \psi=e \psi, \\
e & = \pm \sqrt{p^{2} c^{2}+m^{2} c^{4}} .
\end{aligned}
$$

where $\boldsymbol{h}_{0}$ is the Hamiltonian for a particle with energy $e$ and wave function $\psi$. The Dirac matrices $\boldsymbol{\alpha}$ are given in terms of the Pauli spin matrices $\boldsymbol{\sigma}$ so that

$$
\boldsymbol{\alpha}=\left(\begin{array}{cc}
0 & \boldsymbol{\sigma} \\
\boldsymbol{\sigma} & 0
\end{array}\right), \beta=\left(\begin{array}{cc}
1 & 0 \\
0 & -1
\end{array}\right)
$$

For a free electron and positron the two-body Dirac Hamiltonian $\boldsymbol{H}_{0}$ is the sum of the individual Hamiltonians. The two-body Dirac equation becomes

$$
\boldsymbol{H}_{0} \Psi=\left(\boldsymbol{h}_{0}^{e}+\boldsymbol{h}_{0}^{p}\right) \Psi=E \Psi
$$

or

$$
\boldsymbol{H}_{0} \Psi=\left(c \boldsymbol{\alpha}_{e} \cdot \boldsymbol{p}_{e}+m c^{2} \beta_{e}+c \boldsymbol{\alpha}_{p} \cdot \boldsymbol{p}_{p}+m c^{2} \beta_{p}\right) \Psi=E \Psi .
$$


The total energy $E$ is given by the sum of the individual energies for the electron and positron for which there are four possibilities,

$$
E_{ \pm \pm}=e_{e}+e_{p}= \pm \sqrt{p_{e}^{2} c^{2}+m^{2} c^{4}} \pm \sqrt{p_{p}^{2} c^{2}+m^{2} c^{4}}
$$

The total wave function $\Psi$ is the direct product of the individual wave functions,

$$
\Psi=\psi_{e}\left(\boldsymbol{r}_{e}\right) \times \psi_{p}\left(\boldsymbol{r}_{p}\right)
$$

This direct product wave function has four Dirac components which may be written in several ways:

$$
\begin{aligned}
\Psi= & \left(\begin{array}{c}
\psi_{1}\left(\boldsymbol{r}_{e}\right) \\
\psi_{2}\left(\boldsymbol{r}_{e}\right)
\end{array}\right) \times\left(\begin{array}{c}
\psi_{1}\left(\boldsymbol{r}_{p}\right) \\
\psi_{2}\left(\boldsymbol{r}_{p}\right)
\end{array}\right)=\left(\begin{array}{c}
\psi_{1}\left(\boldsymbol{r}_{e}\right) \psi_{1}\left(\boldsymbol{r}_{p}\right) \\
\psi_{1}\left(\boldsymbol{r}_{e}\right) \psi_{2}\left(\boldsymbol{r}_{p}\right) \\
\psi_{2}\left(\boldsymbol{r}_{e}\right) \psi_{1}\left(\boldsymbol{r}_{p}\right) \\
\psi_{2}\left(\boldsymbol{r}_{e}\right) \psi_{2}\left(\boldsymbol{r}_{p}\right)
\end{array}\right) \equiv\left(\begin{array}{c}
\Psi_{11} \\
\Psi_{12} \\
\Psi_{21} \\
\Psi_{22}
\end{array}\right), \\
\equiv & \Psi_{11} \boldsymbol{e}_{11}+\Psi_{12} \boldsymbol{e}_{12}+\Psi_{21} \boldsymbol{e}_{21}+\Psi_{22} \boldsymbol{e}_{22}, \\
\equiv & \frac{1}{2}\left\{\left(\Psi_{11}+\Psi_{22}\right)\left(\boldsymbol{e}_{11}+\boldsymbol{e}_{22}\right)+\left(\Psi_{11}-\Psi_{22}\right)\left(\boldsymbol{e}_{11}-\boldsymbol{e}_{22}\right)\right. \\
& \left.\quad+\left(\Psi_{12}+\Psi_{21}\right)\left(\boldsymbol{e}_{12}+\boldsymbol{e}_{21}\right)+\left(\Psi_{12}-\Psi_{21}\right)\left(\boldsymbol{e}_{12}-\boldsymbol{e}_{21}\right)\right\} .
\end{aligned}
$$

Here, the latter symmetrized basis is preferred because it has well defined charge-conjugation and inversion symmetries. With this convention for the Dirac components $\Psi_{i j}$ of the Dirac vectors $\boldsymbol{e}_{i j}$, the $i=1,2$ signifies the electron components with positive and negative rest mass, respectively, and the $j=1,2$ signifies the positron components with positive and negative rest mass, respectively, such that

$$
\begin{aligned}
& m c^{2} \beta_{e} \Psi=m c^{2}\left(\Psi_{11} \boldsymbol{e}_{11}+\Psi_{12} \boldsymbol{e}_{12}-\Psi_{21} \boldsymbol{e}_{21}-\Psi_{22} \boldsymbol{e}_{22}\right) \\
& m c^{2} \beta_{p} \Psi=m c^{2}\left(\Psi_{11} \boldsymbol{e}_{11}-\Psi_{12} \boldsymbol{e}_{12}+\Psi_{21} \boldsymbol{e}_{21}-\Psi_{22} \boldsymbol{e}_{22}\right)
\end{aligned}
$$

When the electron and positron interact with a Coulomb potential, it is useful to transform to the relative coordinates,

$$
\boldsymbol{\rho}=\boldsymbol{r}_{e}-\boldsymbol{r}_{p}, \quad \boldsymbol{R}=\frac{1}{2}\left(\boldsymbol{r}_{e}+\boldsymbol{r}_{p}\right)
$$

and their conjugate momenta

$$
\boldsymbol{\pi} \equiv-i \hbar \boldsymbol{\nabla}_{\rho}=\frac{1}{2}\left(\boldsymbol{p}_{e}-\boldsymbol{p}_{p}\right), \quad \boldsymbol{P} \equiv-i \hbar \boldsymbol{\nabla}_{R}=\boldsymbol{p}_{e}+\boldsymbol{p}_{p}
$$


Letting the total momentum be zero, $\boldsymbol{P}=\mathbf{0}$, corresponding to the center of momentum frame, one finds

$$
\boldsymbol{p}_{e}+\boldsymbol{p}_{p}=\mathbf{0}, \quad \boldsymbol{p}_{e}=-\boldsymbol{p}_{p}=\boldsymbol{\pi}
$$

and the two-body Dirac equation (2) for free particles becomes

$$
\boldsymbol{H}_{0} \Psi=\left(c \boldsymbol{\alpha}_{e} \cdot \boldsymbol{\pi}+m c^{2} \beta_{e}-c \boldsymbol{\alpha}_{p} \cdot \boldsymbol{\pi}+m c^{2} \beta_{p}\right) \Psi=E \Psi
$$

From (31) the free particle energies are then,

$$
E_{++}=+2 e_{0}, E_{--}=-2 e_{0}, \quad E_{+-}=E_{-+}=0
$$

where

$$
e_{0}=\sqrt{\pi^{2} c^{2}+m^{2} c^{4}}
$$

The Dirac free particle states $\Psi_{++}$and $\Psi_{--}$, corresponding to energies $E_{++}$and $E_{--}$, respectively, comprise the particle and antiparticle states of 'atomic' positronium for the Dirac 'hole' theory. The free particle states $\Psi_{+-}$and $\Psi_{-+}$, corresponding to energy $E=0$, are degenerate for all relative momentum $\pi$ and give rise to the 'anomalous' states. Note that this degeneracy only occurs for equal masses where $m_{e}=m_{p}=m$ and not for hydrogenic atoms in general. These anomalous degenerate states will be strongly coupled by the electric and magnetic potential which will split their degeneracy. Thus the weak electromagnetic potential has a very strong effect on these degenerate states. One of the main results of this paper is to explicitly find the energies and wave functions of the anomalous states resulting from the splitting of this degeneracy and to calculate the interaction of these anomalous states with the atomic ground-state. Indeed, as explained in the next section, it is this interaction which explains the unusual behavior of the Dirac ground-state near the origin.

For a Coulomb potential, $V_{C}(\rho)$, it is useful to use spherical coordinates, $\boldsymbol{\rho} \equiv(\rho, \theta, \varphi)$, where

$$
V_{C}(\rho)=-e^{2} / \rho
$$

and the two-body Dirac equation (7) becomes

$$
\left(c \boldsymbol{\alpha}_{e} \cdot \boldsymbol{\pi}+m c^{2} \beta_{e}-c \boldsymbol{\alpha}_{p} \cdot \boldsymbol{\pi}+m c^{2} \beta_{p}\right) \Psi=\left(E+e^{2} / \rho\right) \Psi
$$


One can couple the individual spin functions $\chi_{\sigma_{e}}^{\frac{1}{2}}$ and $\chi_{\sigma_{p}}^{\frac{1}{2}}$ of the electron and positron to find functions of total spin $S$ and projection $S_{z}=\Sigma$ such that

$$
\Omega_{\Sigma}^{S} \equiv\left[\chi_{e}^{\frac{1}{2}} \chi_{p}^{\frac{1}{2}}\right]_{\Sigma}^{S}=\sum_{\sigma_{e}, \sigma_{p}} C_{\sigma_{e}}^{\frac{1}{2}} \underset{\sigma_{p}}{\frac{1}{2}}{ }_{\Sigma}^{S} \chi_{\sigma_{e}}^{\frac{1}{2}} \chi_{\sigma_{p}}^{\frac{1}{2}},
$$

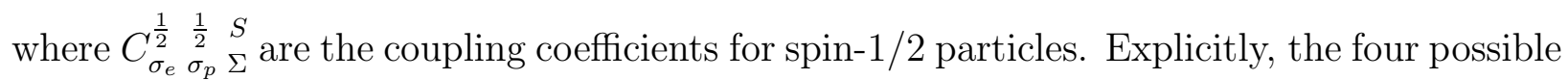
spin functions $\Omega_{\Sigma}^{S}$ for $S=0$ and $S=1$ are

$$
\begin{aligned}
\Omega_{0}^{0} & \equiv\left[\chi_{e}^{\frac{1}{2}} \chi_{p}^{\frac{1}{2}}\right]_{0}^{0}=\left[\chi_{\frac{1}{2}}^{\frac{1}{2}} \chi_{-\frac{1}{2}}^{\frac{1}{2}}-\chi_{-\frac{1}{2}}^{\frac{1}{2}} \chi_{\frac{1}{2}}^{\frac{1}{2}}\right] / \sqrt{2}, \\
\Omega_{0}^{1} & \equiv\left[\chi_{e}^{\frac{1}{2}} \chi_{p}^{\frac{1}{2}}\right]_{0}^{1}=\left[\chi_{\frac{1}{2}}^{\frac{1}{2}} \chi_{-\frac{1}{2}}^{\frac{1}{2}}+\chi_{-\frac{1}{2}}^{\frac{1}{2}} \chi_{\frac{1}{2}}^{\frac{1}{2}}\right] / \sqrt{2}, \\
\Omega_{-1}^{1} & \equiv\left[\chi_{e}^{\frac{1}{2}} \chi_{p}^{\frac{1}{2}}\right]_{-1}^{1}=\chi_{-\frac{1}{2}}^{\frac{1}{2}} \chi_{-\frac{1}{2}}^{\frac{1}{2}} \\
\Omega_{1}^{1} & \equiv\left[\chi_{e}^{\frac{1}{2}} \chi_{p}^{\frac{1}{2}}\right]_{1}^{1}=\chi_{\frac{1}{2}}^{\frac{1}{2}} \chi_{\frac{1}{2}}^{\frac{1}{2}}
\end{aligned}
$$

Using the exchange symmetry of the spin functions, one finds

$$
\left[\chi_{e}^{\frac{1}{2}} \chi_{p}^{\frac{1}{2}}\right]_{\Sigma}^{S}=(-1)^{S+1}\left[\chi_{p}^{\frac{1}{2}} \chi_{e}^{\frac{1}{2}}\right]_{\Sigma}^{S}
$$

So, under particle exchange, the $S=0$ spin functions are antisymmetric and the $S=1$ spin functions are symmetric.

Because the total angular momentum $J$ is conserved for a Coulomb potential, the spherical harmonics for orbital angular momentum $Y_{M}^{L}(\theta, \varphi)$ are coupled with the total spin functions to obtain eigenfunctions of total angular momentum $J$ and projection $J_{z}=N$ where

$$
\left[Y^{L}(\theta, \varphi) \Omega^{S}\right]_{N}^{J}=\sum_{M, \Sigma} C_{M}^{L} \underset{\Sigma}{S}{ }_{N}^{J} Y_{M}^{L}(\theta, \varphi) \Omega_{\Sigma}^{S}
$$

There are four possible angular momentum states $\left[Y^{L} \Omega^{S}\right]_{N}^{J}$ for a given $J$ and $N$ depending on the spin $S$ and the orbital angular momentum $L$, namely,

$$
\begin{gathered}
{\left[Y^{J+1} \Omega^{1}\right]_{N}^{J}=\left[Y^{J+1}\left[\chi_{e}^{\frac{1}{2}} \chi_{p}^{\frac{1}{2}}\right]^{1}\right]_{N}^{J}=\left[\left[Y^{J+1} \chi_{e}^{\frac{1}{2}}\right]^{J+\frac{1}{2}} \chi_{p}^{\frac{1}{2}}\right]_{N}^{J},} \\
{\left[Y^{J-1} \Omega^{1}\right]_{N}^{J}=\left[Y^{J-1}\left[\chi_{e}^{\frac{1}{2}} \chi_{p}^{\frac{1}{2}}\right]^{1}\right]_{N}^{J}=\left[\left[Y^{J-1} \chi_{e}^{\frac{1}{2}}\right]^{J-\frac{1}{2}} \chi_{p}^{\frac{1}{2}}\right]_{N}^{J} .} \\
{\left[Y^{J} \Omega^{0}\right]_{N}^{J}=\left[Y^{J}\left[\chi_{e}^{\frac{1}{2}} \chi_{p}^{\frac{1}{2}}\right]^{0}\right]_{N}^{J}=a\left[\left[Y^{J} \chi_{e}^{\frac{1}{2}}\right]^{J+\frac{1}{2}} \chi_{p}^{\frac{1}{2}}\right]_{N}^{J}-b\left[\left[Y^{J} \chi_{e}^{\frac{1}{2}}\right]^{J-\frac{1}{2}} \chi_{p}^{\frac{1}{2}}\right]_{N}^{J},} \\
{\left[Y^{J} \Omega^{1}\right]_{N}^{J}=\left[Y^{J}\left[\chi_{e}^{\frac{1}{2}} \chi_{p}^{\frac{1}{2}}\right]^{1}\right]_{N}^{J}=b\left[\left[Y^{J} \chi_{e}^{\frac{1}{2}}\right]^{J+\frac{1}{2}} \chi_{p}^{\frac{1}{2}}\right]_{N}^{J}+a\left[\left[Y^{J} \chi_{e}^{\frac{1}{2}}\right]^{J-\frac{1}{2}} \chi_{p}^{\frac{1}{2}}\right]_{N}^{J},}
\end{gathered}
$$


where

$$
a=\sqrt{\frac{J+1}{2 J+1}}, \quad b=\sqrt{\frac{J}{2 J+1}}
$$

are the angular momentum recoupling coefficients for spin- $1 / 2$ particles. Making use of the spin exchange symmetry (13), it follows that we can also write (15) as

$$
\begin{aligned}
{\left[Y^{J+1} \Omega^{1}\right]_{N}^{J} } & =\left[\left[Y^{J+1} \chi_{p}^{\frac{1}{2}}\right]^{J+\frac{1}{2}} \chi_{e}^{\frac{1}{2}}\right]_{N}^{J}, \\
{\left[Y^{J-1} \Omega^{1}\right]_{N}^{J} } & =\left[\left[Y^{J-1} \chi_{p}^{\frac{1}{2}}\right]^{J-\frac{1}{2}} \chi_{e}^{\frac{1}{2}}\right]_{N}^{J}, \\
{\left[Y^{J} \Omega^{0}\right]_{N}^{J} } & =-a\left[\left[Y^{J} \chi_{p}^{\frac{1}{2}}\right]^{J+\frac{1}{2}} \chi_{e}^{\frac{1}{2}}\right]_{N}^{J}+b\left[\left[Y^{J} \chi_{p}^{\frac{1}{2}}\right]^{J-\frac{1}{2}} \chi_{e}^{\frac{1}{2}}\right]_{N}^{J}, \\
{\left[Y^{J} \Omega^{1}\right]_{N}^{J} } & =b\left[\left[Y^{J} \chi_{p}^{\frac{1}{2}}\right]^{J+\frac{1}{2}} \chi_{e}^{\frac{1}{2}}\right]_{N}^{J}+a\left[\left[Y^{J} \chi_{p}^{\frac{1}{2}}\right]^{J-\frac{1}{2}} \chi_{e}^{\frac{1}{2}}\right]_{N}^{J} .
\end{aligned}
$$

\section{A. Coordinate Representation}

In the coordinate representation the single particle operator $\boldsymbol{\sigma} \cdot \boldsymbol{\pi}$ acting on the radial functions $y(\rho)$ is given by [2], [5]

$$
\begin{aligned}
& \boldsymbol{\sigma} \cdot \boldsymbol{\pi} \frac{y}{\rho}\left[Y^{J} \chi^{\frac{1}{2}}\right]_{N}^{J-\frac{1}{2}}=\frac{i \hbar}{\rho}\left(\frac{d y}{d \rho}+\frac{J y}{\rho}\right)\left[Y^{J-1} \chi^{\frac{1}{2}}\right]_{N}^{J-\frac{1}{2}}, \\
& \boldsymbol{\sigma} \cdot \boldsymbol{\pi} \frac{y}{\rho}\left[Y^{J} \chi^{\frac{1}{2}}\right]_{N}^{J+\frac{1}{2}}=\frac{i \hbar}{\rho}\left(\frac{d y}{d \rho}-\frac{(J+1) y}{\rho}\right)\left[Y^{J+1} \chi^{\frac{1}{2}}\right]_{N}^{J+\frac{1}{2}} .
\end{aligned}
$$

Using the recoupling (15)-(17) and the single particle operator equations (18), it is now a simple matter to evaluate $\boldsymbol{\alpha}_{e} \cdot \boldsymbol{\pi}$ and $-\boldsymbol{\alpha}_{p} \cdot \boldsymbol{\pi}$ in (11) and to derive the two-body Dirac partial differential equations in the coordinate representation. Note that $y(\rho)$ satisfies the boundary condition $y(\rho)=0$. The resulting three sets of equations for free particles are given in Appendix A. These equations agree with previous works [4], [1] where cases 1A, $2 \mathrm{~A}$, and $3 \mathrm{~A}$ correspond to the sets 1,3 , and 2 in Ref. [1].

\section{B. Momentum Representation}

Calculations can also be performed in the momentum representation formed by 'Fourier analysis' of the equations in the coordinate representation using spherical Bessel functions $N_{J m} j_{J}\left(k_{m} \rho\right)$ where $\pi=\hbar k_{m}$. The $k_{m}$ are determined by the boundary condition

$$
j_{J}\left(k_{m} \rho_{0}\right)=0, \text { for } m=1,2, \ldots,
$$


with $\rho_{0} \gg 2 n a_{0}$ (where $a_{0}$ is the Bohr radius). The normalizations $N_{J m}$ are determined by

$$
N_{J m} \int_{0}^{\rho_{0}} \rho^{2} j_{J}^{2}\left(k_{m} \rho\right) d \rho=1 .
$$

The Bessel functions $j_{L}\left(k_{m} \rho\right)$ for different $L=J-1, J, J+1$ have the same normalization $N_{J m}$ independent of $L$ given by

$$
N_{J m}=\sqrt{\frac{2}{\rho_{0}^{3} j_{J \pm 1}^{2}\left(k_{m} \rho_{0}\right)}},
$$

where $j_{J+1}\left(k_{m} \rho_{0}\right)=-j_{J-1}\left(k_{m} \rho_{0}\right)$. For high $m \gg 1$ where $k_{m} \rho_{0} \gg 1$ one has the approximations

$$
\begin{aligned}
N_{J m} & \simeq k_{m} \sqrt{2 / \rho_{0}}, \\
k_{m} & \simeq\left(\frac{1}{2} J \pi+m \pi\right) / \rho_{0} .
\end{aligned}
$$

The functions $\rho j_{L}\left(k_{m} \rho\right)$ form an orthonormal set such that

$$
N_{J m}^{2} \int_{0}^{\rho_{0}} \rho^{2} j_{L}\left(k_{m} \rho\right) j_{L}\left(k_{n} \rho\right) d \rho=\delta_{m n} \text { for } L=J-1, J, J+1,
$$

where the $k_{m}$ are, again, determined solely by (19) (for $\left.L=J\right)$.

As in the coordinate basis (15), there are four different momentum bases $|L, S, k\rangle$ for a given $J, N$, and $k$ :

$$
\begin{aligned}
|J+1,1, k\rangle & =i N_{J k} \rho j_{J+1}(k \rho)\left[Y^{J+1} \Omega^{1}\right]_{N}^{J}, \\
|J-1,1, k\rangle & =i N_{J k} \rho j_{J-1}(k \rho)\left[Y^{J-1} \Omega^{1}\right]_{N}^{J} . \\
|J, 0, k\rangle & =N_{J k} \rho j_{J}(k \rho)\left[Y^{J} \Omega^{0}\right]_{N}^{J}, \\
|J, 1, k\rangle & =N_{J k} \rho j_{J}(k \rho)\left[Y^{J} \Omega^{1}\right]_{N}^{J} .
\end{aligned}
$$

Note that the scale factor $\rho$ for spherical coordinates is used in the definition of the wave function as it was in the coordinate representation (18) so that these wave functions are also zero at $\rho=0$. When recoupling, it is useful to define linear combinations of $|J+1,1, k\rangle$ and $|J-1,1, k\rangle$, namely,

$$
\begin{aligned}
& |J \alpha, 1, k\rangle \equiv a|J+1,1, k\rangle+b|J-1,1, k\rangle, \\
& |J \beta, 1, k\rangle \equiv-b|J+1,1, k\rangle+a|J-1,1, k\rangle,
\end{aligned}
$$


where $a$ and $b$ are the recoupling coefficients given in (16). Using the one-particle equations for the spherical Bessel functions, one can find the equivalent equations to (18) for the momentum representation:

$$
\begin{aligned}
\boldsymbol{\sigma} \cdot \boldsymbol{\pi}\left\{j_{J}(k \rho)\left[\left[Y^{J} \chi^{\frac{1}{2}}\right]_{N}^{J-\frac{1}{2}}\right\}\right. & =i \hbar k\left\{j_{J-1}(k \rho)\left[\left[Y^{J-1} \chi^{\frac{1}{2}}\right]_{N}^{J-\frac{1}{2}}\right\}\right. \\
\boldsymbol{\sigma} \cdot \boldsymbol{\pi}\left\{j_{J}(k \rho)\left[\left[Y^{J} \chi^{\frac{1}{2}}\right]_{N}^{J+\frac{1}{2}}\right\}\right. & =-i \hbar k\left\{j_{J+1}(k \rho)\left[\left[Y^{J+1} \chi^{\frac{1}{2}}\right]_{N}^{J+\frac{1}{2}}\right\} .\right.
\end{aligned}
$$

Recoupling the angular wave functions as in (15)-(17), one can evaluate $\boldsymbol{\sigma}_{e} \cdot \pi$ and $-\boldsymbol{\sigma}_{p} \cdot \pi$ in (11) when operating on the wave functions in (20). The three sets of equations for free particles in momentum space in cases $1 \mathrm{~B}, 2 \mathrm{~B}$, and 3B, analogous to cases $1 \mathrm{~A}, 2 \mathrm{~A}$, and $3 \mathrm{~A}$, can now be derived and solved analytically as shown in Appendix B. The free particle wave functions in (20), (21) may also be expanded in terms of products of their single particle components $g_{n_{e}}^{\ell_{e} j_{e}}\left(k r_{e}, \theta_{e}, \varphi_{e}\right) g_{n_{p}}^{\ell_{p} j_{p}}\left(k r_{p}, \theta_{p}, \varphi_{p}\right)$, where

$$
g_{n}^{\ell j}(k r, \theta, \varphi) \equiv j_{\ell}(k r)\left[Y^{\ell}(\theta, \varphi) \chi^{\frac{1}{2}}\right]_{n}^{j},
$$

as shown in Appendix B.

\section{DIRAC AND PAULI SOLUTIONS FOR POSITRONIUM}

For a given case of angular momentum states $\left[Y^{L} \Omega^{S}\right]^{J}$ shown in Appendix B, the Dirac solutions with a Coulomb potential arise from combinations of the four free particle basis $\Psi_{++}, \Psi_{--}, \Psi_{S}, \Psi_{A}$ which have the same $C$ parity from charge-conjugation symmetry and $P$ parity from inversion symmetry as shown by Malenfant [4]. Two are the atomic solutions which are corresponding to the $\Psi_{++}$and $\Psi_{--}$states. Indeed, the $\Psi_{++}$states correspond to the Pauli atomic states of positronium and the $\Psi_{--}$states correspond to the Pauli atomic antiparticle states with negative-energy. The remaining two symmetrized states $\Psi_{S}=\left(\Psi_{+-}+\right.$ $\left.\Psi_{-+}\right) / \sqrt{2}$ and $\Psi_{A}=\left(\Psi_{+-}-\Psi_{-+}\right) / \sqrt{2}$ correspond to the anomalous states.

\section{A. Coulomb Potential}

Appendix A and B are equations for free two-particle Dirac wave functions in the relative coordinates. For a Coulomb potential (10), changes must be made to the equations in Appendices A and B. In Appendix A one must change $E$ to $E-V_{C}=E+e^{2} / \rho$. In Appendix 
B one must change $E$ to $E \delta_{k k^{\prime}}-V_{k k^{\prime}}^{i}$ using the appropriate combination of orthonormal spherical Bessel functions $j_{L}(k \rho)$ to evaluate the matrix elements $V_{k k^{\prime}}^{i}$ as described in this Appendix, depending on the case 1B, 2B, or $3 \mathrm{~B}$.

\section{Atomic States}

In this section, the finite element calculations for the Dirac energies $E_{D}$ of [1] in the coordinate representation are compared to the analytic results of the Pauli approximation $E_{P}$, valid to order $m c^{2} \alpha^{4}$ for the Coulomb energies of the positronium atom. The Pauli basis only includes the $\Psi_{++}$free particle states as shown in Appendix B. The development of Bethe and Salpeter [2] is used for the Coulomb energies $E_{P}$ of the Pauli approximation adopting their notation. While Bethe and Salpeter give the results for both the Coulomb and the Breit interaction together (as derived previously by Ferrell [6]), one can readily extract only the Coulomb part from their results. Accordingly, one has the following formulas for the Pauli approximation to the Coulomb energies $E_{P}$ for a given $(n L S J)$ state of positronium:

$$
E_{P}=2 m c^{2}+H_{0}+H_{1}+H_{3}^{C}+H_{4}^{C} .
$$

where

$$
\begin{aligned}
& H_{0}=-\frac{m c^{2} \alpha^{2}}{4 n^{2}} \\
& H_{1}=\frac{3 m c^{2} \alpha^{4}}{64 n^{4}}-\frac{m c^{2} \alpha^{4}}{8 n^{3}(2 L+1)}, \\
& H_{3}^{C}=\frac{m c^{2} \alpha^{4}}{8 n^{3} L(L+1)(2 L+1)}\left\{\begin{array}{cc}
L & J=L+1 \\
-1 & J=L \\
-(L+1) & J=L-1
\end{array}\right\}\left(1-\delta_{L 0}\right) \delta_{S 1}, \\
& H_{4}^{C}=\frac{m c^{2} \alpha^{4}}{8 n^{3}} \delta_{L 0} .
\end{aligned}
$$

The term $H_{0}$ is just the second order positronium energy from the Schrodinger equation and $H_{1}$ is the relativistic kinetic correction to the fourth order. The terms $H_{3}^{C}+H_{4}^{C}$ correspond to the fine structure corrections of positronium for a Coulomb potential to the fourth order. Note the Kronecker deltas are such that $H_{3}^{C}=0$ if $L=0$ or $S=0$. Also, note that $H_{4}^{C}$ cancels the second term of $H_{1}$ when $L=0$. Later, the magnetic terms $H_{2}^{B}, H_{3}^{B}$, and $H_{5}^{B}$ are considered for the Breit potential which also contribute to the fourth order. 
The Dirac energies, $E_{D}-2 m c^{2}$, in Ref. [1] for all $(n L S J)$ states up to $n=3$ are compared with the Pauli energies to fourth order, $E_{P}-2 m c^{2}$, in Table I where cases 1,2,3 correspond to sets 1,3,2 in Ref. [1]. All calculations use the approximate value $\alpha=1 / 137$ as in Ref. [1] with the energies in units of Hartree $=m c^{2} \alpha^{2}$ and the differences in energies $E_{D}-E_{P}$ is in units of $M h z$ using the conversion Hartree $=\left(m c^{2} \alpha^{2} / h\right) H z=6.579684 \times 10^{9}$ $M H z$. The agreement in Table 1 between $E_{D}$ and $E_{P}$ is surprising. It has been shown by Ishidzu [7, 8] that the energies for the atomic states for the two-body Dirac equation in a Coulomb potential can be expanded in a power series in $\alpha^{2}$. Thus, according to Ishidzu, the energy differences $E_{D}-E_{P}$ in Table 1 should be of order $m c^{2} \alpha^{6} / h \sim 20 \mathrm{MHz}$ which is approximately the agreement found for the $L=0$ states.

Actually, one can fit the energy differences $E_{D}-E_{P}$ for the three $L=0$ states $(n 000)$ to the expression $\nu_{0} / n^{3}$ with $\nu_{0}=-10.6376 \mathrm{Mhz}$ and a standard deviation of only $\sigma=0.5 \mathrm{Khz}$. Similarly, one can fit the energy differences $E_{D}-E_{P}$ for the three $L=0$ states $(n 011)$ to the expression $\nu_{1} / n^{3}$ with $\nu_{1}=-7.2724 M h z$ and a standard deviation of $\sigma=8 \mathrm{Khz}$. The $L=1$ states have accuracies of $\sim 10 \mathrm{kHz}$ or less and the $L=2$ states have accuracies of $\sim 1 k H z$ or less. The overall standard deviation for all $18(n L S J)$ states is then only $\sigma=7 \mathrm{Khz}$ using the constants $\nu_{0}$ and $\nu_{1}$. Altogether, this remarkable agreement is proof of the accuracy of the calculations in Ref. [1] and corresponds to 16 significant figures with respect to the positronium rest mass $2 m c^{2}$. Note that this numerical accuracy is much greater than either the theoretical accuracy of QED calculations for atomic positronium, presently to order $m c^{2} \alpha^{6}$, or the experimental error in any positronium spectroscopy, both of which are typically of $\sim 1 \mathrm{MHz}$ accuracy.

However, this numerical accuracy in the energy differences $E_{D}-E_{P}$ is somewhat misleading in that the Dirac equation with a Coulomb potential is missing all terms $m c^{2} \alpha^{n}$ of odd order $n$ [7, 8], the largest of which is $m c^{2} \alpha^{5}$, and $E_{P}$ is only calculated to order $m c^{2} \alpha^{4}$. In fact, it has been shown by Fulton and Martin [9] that there is an energy shift for the $L=0$ states of positronium, due to a Coulomb term of order $m c^{2} \alpha^{5}$ in the Pauli approximation, given approximately by

$$
\Delta E_{L=0} \simeq \frac{-m c^{2} \alpha^{5}}{8 n^{3}}=-\frac{320}{n^{3}} M h z .
$$

Thus, for a Coulomb potential, the Pauli energy of the ground-state is approximately $320 M h z$ lower than the Dirac energy. There are many more terms of this order due to QED corrections for the $(n L S J)$ states as treated in Ref. [9]. Finally, there are the mag- 


\begin{tabular}{|c|c|c|c|c|}
\hline$n L S S$ & Case & $E_{D}-2 m c^{2}$ (Hartree) & $E_{P}-2 m c^{2}$ (Hartree) & $E_{D}-E_{P}(M H z)$ \\
\hline $\begin{array}{llll}1 & 0 & 0 & 0\end{array}$ & 1 & -0.24999750414752 & -0.24999750253077 & -10.6377 \\
\hline $\begin{array}{llll}1 & 0 & 1 & 1\end{array}$ & 3 & -0.24999750363633 & -0.24999750253077 & -7.2742 \\
\hline 2000 & 1 & -0.06249984411014 & -0.06249984390817 & -1.3289 \\
\hline 2011 & 3 & -0.06249984404437 & $\begin{array}{lllll}-0.062 & 499 & 843908 & 17\end{array}$ & -0.8962 \\
\hline 2101 & 1 & -0.06250012140376 & -0.06250012140475 & 0.0066 \\
\hline 21110 & 3 & -0.06250039890481 & -0.06250039890133 & -0.0229 \\
\hline 2111 & 2 & -0.06250026015303 & -0.06250026015304 & 0.0001 \\
\hline 21112 & 3 & -0.06249998265670 & -0.06249998265646 & -0.0016 \\
\hline $\begin{array}{llll}3 & 0 & 0 & 0\end{array}$ & 1 & -0.027 77774700471 & -0.02777774694482 & -0.3941 \\
\hline $\begin{array}{llll}3 & 0 & 1 & 1\end{array}$ & 3 & -0.027 77774698512 & -0.02777774694482 & -0.2651 \\
\hline $\begin{array}{llll}3 & 1 & 0 & 1\end{array}$ & 1 & -0.02777782916571 & -0.02777782916603 & 0.0021 \\
\hline 31110 & 3 & -0.02777791138816 & -0.02777791138724 & -0.0060 \\
\hline 3111 & 2 & -0.02777787027658 & -0.02777787027664 & 0.0004 \\
\hline 31112 & 3 & -0.02777778805552 & -0.02777778805543 & -0.0006 \\
\hline 3202 & 1 & -0.027 77779627754 & -0.027 77779627755 & 0.0001 \\
\hline 3211 & 3 & -0.02777782094403 & -0.027 77782094391 & -0.0007 \\
\hline 3212 & 2 & -0.02777780449968 & -0.02777780449967 & -0.0001 \\
\hline 32213 & 3 & -0.027 77777983331 & -0.027 77777983331 & -0.0000 \\
\hline
\end{tabular}

TABLE I: Comparison of Two-Body Dirac Energies of Scott, et al. with the Pauli Energies for a Coulomb Potential

netic terms of order $m c^{2} \alpha^{4}$, considered below, which contribute to the fine structure.

The Dirac radial components of the ground-state wave function, shown in Fig. 1, are calculated in the coordinate representation using equations (A2) for Case 1A. As shown in Ref. [1], accurate ground-state wave functions for the Dirac equation can only be achieved with a grid which has elements in the region of $\rho \sim 1$ Fermi. In this work, a grid is used to cover the three Regions shown in Fig. 1: Region 1, with 10 equal elements of $\Delta \rho=10^{-5}$ Bohr, Region 2, with 9 equal elements of $\Delta \rho=0.002$ Bohr and Region 3, with 79 equal elements of $\Delta \rho=0.5$ Bohr for $n=1$ states. The endpoint and element size of this last 
region varies proportionately with $n$ of the positronium radius $a=2 n$ Bohr. With five point Lagrangian interpolations, the 100 finite elements correspond to 399 grid points. In atomic units, Region 1 is near the classical radius of the electron or positron $\rho=\alpha^{2} \sim(1 / 137)^{2}$ Bohr. Region 2 is near the Compton wavelength $\rho=\alpha \sim(1 / 137)$ Bohr and Region 3 is near the radius $\rho=2 n$ Bohr of positronium. In this figure, a log-log scale is used to investigate the wave function at very small $\rho$. The large dots in the figure correspond to the grid points used for the three different regions in the Dirac calculations. For five point Lagrangian interpolation, there are four grid points for every element. These dots merge into a continuous line when the spacing becomes small on this log scale.

The Pauli wave functions, calculated using the $\Psi_{++}$basis in Appendix B, are also shown in Fig. 1. One might expect very good agreement between the Dirac and Pauli wave functions as is the case for their energies. However, the Dirac solution for $y_{11}^{2}$ and $y_{22}^{2}$ differ greatly from their Pauli solutions in Region 1. This unusual behavior is the result of the coupling of atomic and anomalous states by the Coulomb potential. As shown in Fig. 1, the Dirac wave function $y_{11}$ for the positronium ground-state is approximately the Schrodinger ground-state wave function $y_{S}$ such that $y_{11}^{2} \simeq y_{S}^{2}=\frac{1}{2} \rho^{2} e^{-\rho}$ in atomic units. Also shown in Fig. 1, the Pauli wave function $y_{22}^{2}$ reaches its maximum near Region 2. On the other hand, the Dirac wave function $y_{22}^{2}$ reaches its maximum near Region 1 as has been shown previously in Ref. [1]. In order to explain the unusual behavior of the Dirac ground-state solutions in Region 1, it is necessary to first consider the anomalous states arising from the $\Psi_{+-}$and $\Psi_{-+}$free particle wave functions.

\section{Anomalous States and the Discrete Variable Representation (DVR)}

It is shown below, both numerically and analytically, that there are bound-state solutions of the Dirac equation for the anomalous states. The anomalous states can be obtained most readily in the momentum basis using Appendix B because this basis allows one to clearly distinguish the anomalous states $\Psi_{+-}$and $\Psi_{-+}$from the atomic states $\Psi_{++}$and $\Psi_{--}$. However, first we treat the anomalous states in the coordinate basis.

In the coordinate basis, the finite element calculations using (A2) for the angular momentum states $\left[Y^{0} \Omega^{0}\right]^{0}$ give solutions for both the Dirac atomic bound-states and the Dirac anomalous bound-states simultaneously. The energy solutions for the anomalous states in 
Region 1 are shown in Fig. 2 and their respective wave function solutions are shown in Fig.

3. The solutions shown are bound-states.

As seen in Figs. 2 and 3, the solutions are approximately Dirac delta functions,

$$
\left(y_{11}^{0}-y_{22}^{0}\right) / \sqrt{2} \simeq C \delta\left(\rho-\rho_{i}\right)
$$

with energy $E_{i} \simeq-e^{2} / \rho_{i}$ where $C$ is the normalization and

$$
\rho_{i}=i \Delta \rho
$$

are at the Lagrangian nodes themselves with spacing $\Delta \rho=10^{-4} / 40$. For clarity only every fourth wave function is shown in Fig. 3, corresponding to the vertical lines in Fig. 2 at the element boundaries. These delta functions are approximately the Lagrangian nodal functions themselves although they have been orthogonalized by the diagonalization and are now centered at the nodes. Note that the delta functions in the coordinate bases for the anomalous states are strictly linked to the grid points. This is not an artefact of the numerical calculations in the coordinate bases as demonstrated below using the momentum bases. In Fig. 3 the component $\left(y_{12}^{+}+y_{21}^{+}\right)$is too small to be seen.

Instead of solving the set of equations (A2), these same anomalous bound-state solutions can be obtained more directly by solving the simpler equation in the same finite element coordinate basis for Case 1A corresponding to

$$
\left(y_{11}^{0}-y_{22}^{0}\right)=\frac{2 m c^{2}\left(y_{11}^{0}+y_{22}^{0}\right)}{E+e^{2} / \rho},
$$

where $E$ has been replaced by $E+e^{2} / \rho$. The radial function $\left(y_{11}^{0}-y_{22}^{0}\right)$ is singular near $E=-e^{2} / \rho$ unless $\left(y_{11}^{0}+y_{22}^{0}\right)=0$ in which case one obtains the simple equation

$$
-\left(e^{2} / \rho\right)\left(y_{11}^{0}-y_{22}^{0}\right)=E\left(y_{11}^{0}-y_{22}^{0}\right) .
$$

The anomalous energies and wave functions are found by simply diagonalizing the potential matrix of $V_{C}(\rho)=-e^{2} / \rho$ in the coordinate basis. The resulting radial solutions of (27) are indistinguishable from the solutions shown in Fig. 2 and Fig. 3.

The same delta function solutions for $\left[Y^{0} \Omega^{0}\right]^{0}$ states can also be found using the momentum basis in Case 1B by solving the equations in (B2). In this case one uses normalized spherical Bessel functions $N \rho j_{0}\left(k_{m} \rho\right)=\sqrt{2 / \rho_{0}} \sin \left(k_{m} \rho\right)$ where $m=1,2, \ldots, M$ with $M=40$ 
and $\rho_{0}=10^{-4}$ Bohr to find the solutions in Region 1 corresponding to Fig. 3. The calculated anomalous state wave functions in this basis are shown in Fig. 4. These solutions must be linear combinations of the free particle states $\Psi_{S}^{0}$ in $(\underline{\mathrm{B} 3})$ which have $E=0$. The radial solutions for these $\left[Y^{0} \Omega^{0}\right]^{0}$ anomalous bound-states are also approximate delta functions. We can write the overall wave function as

$$
\left|\Psi_{S}^{0}, i\right\rangle \simeq C \delta\left(\rho-\rho_{i}\right) Y^{0} \Omega^{0}\left(\boldsymbol{e}_{11}-\boldsymbol{e}_{22}\right) / \sqrt{2}
$$

with energy $E_{i} \simeq-e^{2} / \rho_{i}$ where $C$ is the normalization and

$$
\rho_{i}=i \Delta \rho \text { for } i=1,2, \ldots, M-1
$$

as in the case of the coordinate representation (25), (26) where the spacing is now

$$
\Delta \rho=\rho_{0} / M
$$

Again, we note that in Fig. 4 the component $\left(y_{12}^{\alpha}+y_{21}^{\alpha}\right)$ is too small to be seen.

Instead of solving the set of equations ( $(\overline{B 2})$, these same anomalous bound-state solutions can be obtained more directly by solving the equation in the same momentum basis (denoting $k_{m}$ by $m$ ), equivalent to (27):

$$
-\sum_{m^{\prime}}\left\langle e^{2} / \rho\right\rangle_{m m^{\prime}}\left(c_{11}^{0}-c_{22}^{0}\right)_{m^{\prime}}=E\left(c_{11}^{0}-c_{22}^{0}\right)_{m}
$$

where $\left(c_{11}^{0}+c_{22}^{0}\right)_{m}=0$ and $\left\langle-e^{2} / \rho\right\rangle_{m m^{\prime}}=V_{m m^{\prime}}^{0}$ (see Appendix B). Again, the anomalous energies and wave functions are found by simply diagonalizing the potential matrix $V_{m m^{\prime}}^{0}$. The resulting radial solutions of (31) are indistinguishable from the solutions shown in Fig. 4 .

In general, for a finite basis set, in either the coordinate or momentum representation, the solutions to the equation

$$
V(\rho) \psi(\rho)=E \psi(\rho) .
$$

are found to be the approximate delta functions such that

$$
\begin{gathered}
\psi^{\prime}(\rho) \simeq C \delta\left(\rho-\rho^{\prime}\right), \\
E^{\prime} \simeq V\left(\rho^{\prime}\right),
\end{gathered}
$$


with $C$ and the discrete grid $\rho^{\prime}$ to be determined. Such solutions are called the discrete variable representation $(D V R)$ and are found by simply diagonalizing the potential $V(\rho)$ in the chosen basis. They numerically converge to exact delta functions only for an infinite basis for which they form highly localized bound-states. This assumes that the basis set is complete and obeys the completeness relation, which for the spherical Bessel functions is

$$
\frac{2}{\rho_{0}} \sum_{m=1}^{\infty} \rho^{2} j_{L}\left(k_{m} \rho\right) j_{L}\left(k_{m} \rho^{\prime}\right)=\delta\left(\rho-\rho^{\prime}\right) \text { for } L=0,1, \ldots
$$

The DVR wave functions are useful in quantum chemistry because any potential $V(\rho)$ is diagonal in this basis with energy $V\left(\rho^{\prime}\right)$ at the points $\rho^{\prime}$. For any finite basis set, the discrete variable representation forms a discrete grid, similar to a finite element grid, at positions $\rho_{i}$ corresponding to the zeros of the basis functions. The grid then depends on the basis set chosen to form the DVR representation. For a review of the literature see the work of Light and Carrington [10] and the references therein.

For a finite basis set, one can find the normalization $C$ of the approximate delta functions $C \delta\left(\rho-\rho_{i}\right)$ using the property

$$
\int \delta\left(\rho-\rho_{i}\right) d \rho=1
$$

For a delta function $\delta\left(\rho-\rho_{i}\right)$ that has a height $\delta(0)$ and a full-width-at-half-maximum (FWHM) $\Delta \rho$, the integral becomes

$$
\int \delta\left(\rho-\rho_{i}\right) d \rho=\delta(0) \Delta \rho=1
$$

or

$$
\delta(0)=1 / \Delta \rho
$$

The normalization condition on the delta function $C \delta\left(\rho-\rho_{i}\right)$ then becomes

$$
C^{2} \int \delta\left(\rho-\rho_{i}\right)^{2} d \rho=C^{2} \delta(0)^{2} \Delta \rho=1
$$

so that

$$
C=\sqrt{\Delta \rho}
$$


In the momentum basis, the approximated delta functions for the $i$ th eigenfunction $\left(y_{11}^{0}-\right.$ $\left.y_{22}^{0}\right)_{i} / \sqrt{2}$ are given simply by the completeness condition on the radial basis $N \rho j_{0}\left(k_{m} \rho\right)$ in (34) for a finite basis set at the discrete $\rho_{i}$,

$$
\begin{aligned}
\left(y_{11}^{0}-y_{22}^{0}\right)_{i} / \sqrt{2} & =\sqrt{\frac{\rho_{0}}{M}} \frac{2}{\rho_{0}} \sum_{m=1}^{M-1} \sin \left(k_{m} \rho\right) \sin \left(k_{m} \rho_{i}\right), \\
& \simeq \sqrt{\Delta \rho} \delta\left(\rho-\rho_{i}\right)
\end{aligned}
$$

for $i=1,2, \ldots, M-1$. These analytic wave functions for $M=40$ are compared to those wave functions calculated numerically in Fig. 4. Thus, the completeness relation allows us to readily derive these analytic DVR solutions for the momentum basis. One can also show that (38) forms an orthonormal basis set such that

$$
\begin{aligned}
I_{i j} & =\frac{1}{2} \int_{0}^{\rho_{0}} d \rho\left(y_{11}^{0}-y_{22}^{0}\right)_{i}\left(y_{11}^{0}-y_{22}^{0}\right)_{j}, \\
& =\Delta \rho\left(\frac{2}{\rho_{0}}\right)^{2} \int_{0}^{\rho_{0}} d \rho \sum_{m=1}^{M-1} \sum_{m^{\prime}=1}^{M-1} \sin \left(k_{m} \rho\right) \sin \left(k_{m} \rho_{i}\right) \sin \left(k_{m^{\prime}} \rho\right) \sin \left(k_{m^{\prime}} \rho_{j}\right) \\
& =\frac{2}{\sqrt{M \rho_{0}}} \sum_{m=1}^{M-1} \sin \left(k_{m} \rho_{i}\right) \sin \left(k_{m} \rho_{j}\right) \\
& =\delta_{i j} .
\end{aligned}
$$

So far, only the anomalous states $\left|\Psi_{S}^{0}, i\right\rangle$ in (28) for $\left[Y^{0} \Omega^{0}\right]^{0}$ have been considered. However, one obtains the exact same set of equations for case $3 \mathrm{~B}$ states with $\left[Y^{0} \Omega^{0}\right]^{0}$. In particular, for the $\Psi_{A}^{0}$ states in (Bי) one has the equation in (B8) after replacing $E$ with $E+e^{2} / \rho$,

$$
-\sum_{m^{\prime}}\left\langle e^{2} / \rho\right\rangle_{m m^{\prime}}\left(c_{12}^{0}-c_{21}^{0}\right)_{m^{\prime}}=E\left(c_{12}^{0}-c_{21}^{0}\right)_{m}
$$

where $\left(c_{12}^{0}+c_{21}^{0}\right)_{m}=0$ and $\left\langle-e^{2} / \rho\right\rangle_{m m^{\prime}}=V_{m m^{\prime}}^{0}$. The solutions for these case 3 anomalous bound-states are

$$
\left|\Psi_{A}^{0}, i\right\rangle=\sqrt{\Delta \rho} \delta\left(\rho-\rho_{i}\right) Y^{0} \Omega^{0}\left(\boldsymbol{e}_{12}-\boldsymbol{e}_{21}\right) / \sqrt{2},
$$

with energy $E_{i}=-e^{2} / \rho_{i}$. Thus, there are two degenerate solutions $\left|\Psi_{S}^{0}, i\right\rangle$ and $\left|\Psi_{A}^{0}, i\right\rangle$ for the $Y^{0} \Omega^{0}$ anomalous states which have radial wave functions $\sqrt{\Delta \rho} \delta\left(\rho-\rho_{i}\right)$ and energies $-e^{2} / \rho_{i}$.

There are also two anomalous bound-state $J=0$ solutions with $\left[Y^{L} \Omega^{S}\right]^{J}=\left[Y^{1} \Omega^{1}\right]^{0}$

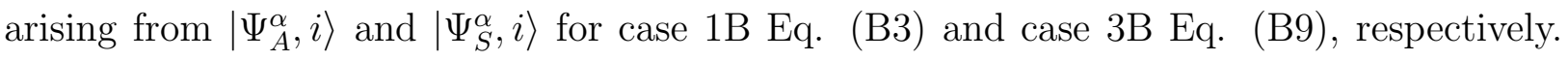


Summarizing these results, one has the four $J=0$ anomalous bound-states which encompass the four different possible $C$ and $P$ parities as denoted in Appendix B:

\begin{tabular}{|c|c|c|c|c|c|}
\hline State & $\psi(\rho)\left[Y^{L} \Omega^{S}\right]^{J}$ & Dirac Vector & Case & $C$ & $P$ \\
\hline$\left|\Psi_{S}^{0}, i\right\rangle$ & $\sqrt{\Delta \rho} \delta\left(\rho-\rho_{i}\right)\left[Y^{0} \Omega^{0}\right]^{0}$ & $\left(\boldsymbol{e}_{11}-\boldsymbol{e}_{22}\right) / \sqrt{2}$ & 1 & 1 & -1 \\
\hline$\left|\Psi_{A}^{0}, i\right\rangle$ & $\sqrt{\Delta \rho} \delta\left(\rho-\rho_{i}\right)\left[Y^{0} \Omega^{0}\right]^{0}$ & $\left(\boldsymbol{e}_{12}-\boldsymbol{e}_{21}\right) / \sqrt{2}$ & 3 & -1 & 1 \\
\hline$\left|\Psi_{A}^{\alpha}, i\right\rangle$ & $\sqrt{\Delta \rho} \delta\left(\rho-\rho_{i}\right)\left[Y^{1} \Omega^{1}\right]^{0}$ & $\left(\boldsymbol{e}_{12}-\boldsymbol{e}_{21}\right) / \sqrt{2}$ & 1 & -1 & -1 \\
\hline$\left|\Psi_{S}^{\alpha}, i\right\rangle$ & $\sqrt{\Delta \rho} \delta\left(\rho-\rho_{i}\right)\left[Y^{1} \Omega^{1}\right]^{0}$ & $\left(\boldsymbol{e}_{11}-\boldsymbol{e}_{22}\right) / \sqrt{2}$ & 3 & 1 & 1 \\
\hline
\end{tabular}

The $\left|\Psi_{S}^{0}, i\right\rangle$ are the only $J=0$ anomalous states which have the same $C$ and $P$ parities as the atomic ground-state, namely $C=1$ and $P=-1$. As a result, they are the only anomalous states which can interact with the atomic ground-state.

\section{Coupling of the Atomic Ground State with Anomalous States}

It can now be shown that the anomalous wave functions $\left|\Psi_{S}^{0}, i\right\rangle$ above for $\left[Y^{0} \Omega^{0}\right]^{0}$ are coupled by the Coulomb potential $V_{C}$ with the zero-order Dirac ground-state atomic wave function $\left|\Psi_{D}\right\rangle^{(0)}$ for $\left[Y^{0} \Omega^{0}\right]^{0}$. This coupling explains the unusual behavior of the Dirac wave functions in Region 1 in Fig. 1. This coupling can be determined analytically and compared to the Dirac wave functions calculated in Ref. [1] which are duplicated in Fig. 1 on a different scale.

The radial Schroedinger ground-state wave function (in atomic units) for positronium is

$$
y_{S}(\rho)=\frac{\rho e^{-\rho / 2}}{\sqrt{2}}
$$

The Dirac ground-state vector to zero order, is then

$$
\left|\Psi_{D}\right\rangle^{(0)} \simeq y_{S} Y^{0} \Omega^{0} \boldsymbol{e}_{11}
$$

The Coulomb coupling of $\left|\Psi_{D}\right\rangle^{(0)}$ with anomalous state $\left|\Psi_{S}^{0}, i\right\rangle$ at $\rho_{i}$ in (41) is given simply by the integral approximation over $\Delta \rho$

$$
\begin{aligned}
\left\langle\Psi_{S}^{0}, i\left|\frac{-e^{2}}{\rho}\right| \Psi_{D}\right\rangle^{(0)} & =\left\langle\sqrt{\Delta \rho / 2} \delta\left(\rho-\rho_{i}\right)\left(\boldsymbol{e}_{11}-\boldsymbol{e}_{22}\right)\left|\frac{-e^{2}}{\rho}\right| y_{S}(\rho) \boldsymbol{e}_{11}\right\rangle \simeq-\sqrt{\Delta \rho / 2} \frac{e^{2}}{\rho_{i}} y_{S}\left(\rho_{i}\right) \delta(0) \Delta \rho, \\
& =-\sqrt{\Delta \rho / 2} \frac{e^{2}}{\rho_{i}} y_{S}\left(\rho_{i}\right),
\end{aligned}
$$


using $\delta(0) \Delta \rho=1$ (36). From first order perturbation theory, the first order correction of the Dirac ground-state $\left|\Psi_{D}\right\rangle$ of energy $E_{D} \simeq 2 m c^{2}$ due to the Coulomb coupling with the anomalous state $\left|\Psi_{S}^{0}, i\right\rangle$ of energy $E_{i}=-e^{2} / \rho_{i}$ is

$$
\begin{aligned}
\left|\Psi_{D}\left(\rho_{i}\right)\right\rangle^{(1)} & =\frac{\left\langle\Psi_{S}^{0}, i\left|\frac{-e^{2}}{\rho}\right| \Psi_{D}\right\rangle^{(0)}}{E_{D}-E_{i}}\left|\Psi_{S}^{0}, i\right\rangle, \\
& =-y_{S}\left(\rho_{i}\right)\left\{\frac{e^{2}}{2 \rho_{i}} \frac{1}{\left(2 m c^{2}+e^{2} / \rho_{i}\right)}\right\} Y^{0} \Omega^{0}\left(\boldsymbol{e}_{11}-\boldsymbol{e}_{22}\right) .
\end{aligned}
$$

where we evaluate $\left|\Psi_{S}^{0}, i\right\rangle$ at $\rho=\rho_{i}$. For an infinite basis set, the DVR grid at $\rho_{i}$ becomes continuous. Letting

$$
g=\frac{e^{2}}{2 \rho_{i}} \frac{1}{\left(2 m c^{2}+e^{2} / \rho_{i}\right)},
$$

the positronium ground-state wave function at $\rho_{i}$ becomes, to first order,

$$
\left|\Psi_{D}\left(\rho_{i}\right)\right\rangle \simeq\left|\Psi_{D}\left(\rho_{i}\right)\right\rangle^{(0)}+\left|\Psi_{D}\left(\rho_{i}\right)\right\rangle^{(1)}=y_{S}\left(\rho_{i}\right)(1-g) Y^{0} \Omega^{0} \boldsymbol{e}_{11}+y\left(\rho_{i}\right) g Y^{0} \Omega^{0} \boldsymbol{e}_{22}
$$

The analytic values of the Dirac radial components are then

$$
y_{11}=y_{S}(1-g), \quad y_{22}=y_{S} g
$$

The square of these Dirac components, $y_{22}^{2}$ and $y_{11}^{2}$, are plotted in Fig. 5. This very simple result (44) explains quite well the unusual behavior of the ground-state Dirac wave function of positronium in Region 1. Note that the analytic values of $y_{22}^{2}$ are not correct in Region 3 where the above approximations fail. Assuming an infinite basis set, the $\rho_{i}$ become continuous $\rho_{i} \rightarrow \rho^{\prime}$ and the Dirac delta functions become exact $\delta\left(\rho-\rho^{\prime}\right)$ (34). In the limit $\rho_{i} \rightarrow 0$, where $e^{2} / \rho_{i} \gg 2 m c^{2}$, it can be seen from (43) that $g \rightarrow \frac{1}{2}$ and these Dirac components (44) become equal to one-half the Schroedinger wave function, $y_{11}=y_{22}=\frac{1}{2} y_{S}$. The fact that the two Dirac components $y_{11}^{2}$ and $y_{22}^{2}$ converge for small $\rho$ can be clearly seen in Region 1 of Fig. 5. In general, for any $(n L S J)=(n 000)$ Schroedinger radial wave function $y_{n}(\rho)$, one can obtain the Dirac radial components $y_{11}$ and $y_{22}$ by replacing $y_{S}$ in (44) with $y_{n}$ where $y_{1}=y_{S}$.

\section{B. Magnetic Potential}

The atomic bound-states and anomalous bound-states behave quite differently in the presence of a magnetic potential $\boldsymbol{V}_{M}$. The two-body Dirac equation is now solved including 
the magnetic potential so that the new Hamiltonian is

$$
\boldsymbol{H}=\boldsymbol{H}_{0}+\boldsymbol{V}_{C}+\boldsymbol{V}_{M}
$$

The form of $\boldsymbol{V}_{M}$ is different for atomic and anomalous states.

\section{Atomic States}

To determine the fine structure of atomic positronium to order $m c^{2} \alpha^{4}$ one must include the magnetic potential. For atomic bound-states the appropriate potential is in the Coulomb gauge. This is because, for atomic states, where $\hbar k \ll m c$, the Dirac operators $\boldsymbol{\alpha}_{e}$ and $\boldsymbol{\alpha}_{p}$ have expectation values close to the fine structure constant $\alpha=e^{2} / \hbar c \sim v / c$. In the Coulomb gauge one obtains the Breit potential [11],

$$
\boldsymbol{V}_{M}=\boldsymbol{V}_{B}(\rho)=\frac{e^{2}}{2 \rho}\left[\boldsymbol{\alpha}_{e} \cdot \boldsymbol{\alpha}_{p}+\left(\boldsymbol{\alpha}_{e} \cdot \hat{\boldsymbol{r}}_{e}\right)\left(\boldsymbol{\alpha}_{p} \cdot \hat{\boldsymbol{r}}_{p}\right)\right],
$$

as result of second order perturbation theory corresponding to the exchange of a transverse photon.

For atomic bound-states of positronium, the Breit potential gives fine structure corrections of order $m c^{2} \alpha^{4}$ which can be determined, as in the case of the Coulomb potential above, using expectation values $\left\langle\boldsymbol{V}_{M}\right\rangle$ in the Pauli approximation. One finds [2], letting $H_{5}^{B}=H_{5}^{B}(1)+H_{5}^{B}(2)$,

$$
\begin{gathered}
H_{2}^{B}=\frac{m c^{2} \alpha^{4}}{8 n^{4}}-\frac{3 m c^{2} \alpha^{4}}{8 n^{3}(2 L+1)}+\frac{m c^{2} \alpha^{4}}{8 n^{3}} \delta_{L 0}, \\
H_{3}^{B}=\frac{2 m c^{2} \alpha^{4}}{8 n^{3} L(L+1)(2 L+1)}\left\{\begin{array}{cc}
L & J=L+1 \\
-1 & J=L \\
-(L+1) & J=L-1
\end{array}\right\}\left(1-\delta_{L 0}\right) \delta_{S 1}, \\
H_{5}^{B}(1)=-\frac{m c^{2} \alpha^{4}}{4 n^{3}} \delta_{S 0} \delta_{L 0}+\frac{m c^{2} \alpha^{4}}{12 n^{3}} \delta_{S 1} \delta_{L 0}, \\
H_{5}^{B}(2)=-\frac{m c^{2} \alpha^{4}}{8 n^{3} L(L+1)(2 L+1)}\left\{\begin{array}{cc}
L /(2 L+3) & J=L+1 \\
(L+1) /(2 L-1) & J=L-1
\end{array}\right\}\left(1-\delta_{L 0}\right) \delta_{S 1} .
\end{gathered}
$$


To this order, there is an additional term due to the energy change resulting from positronium annihilation for $S=1$ states given by

$$
H_{a n}=\frac{m c^{2} \alpha^{4}}{4 n^{3}} \delta_{S 1} \delta_{L 0}
$$

The Pauli energies $E_{p}^{\prime}$ of $\boldsymbol{H}$ including $H_{a n}$ is then,

$$
E_{P}^{\prime}=2 m c^{2}+H_{0}+H_{1}+H_{3}^{C}+H_{4}^{C}+H_{2}^{B}+H_{3}^{B}+H_{5}^{B}+H_{a n} .
$$

Combining terms, one finds to fourth order [6]

$$
\begin{gathered}
E_{P}^{\prime}=2 m c^{2}-\frac{m c^{2} \alpha^{2}}{4 n^{2}}+\frac{m c^{2} \alpha^{4}}{n^{3}}\left\{\frac{11}{64 n}-\frac{1}{2(2 L+1)}+\xi \delta_{S 1}\right\}, \\
\text { where } \xi=\frac{7 \delta_{L 0}}{12}+\frac{1-\delta_{L 0}}{4(2 L+1)}\left\{\begin{array}{cc}
\frac{3 L+4}{(L+1)(2 L+3)} & J=L+1 \\
-\frac{1}{L(L+1)} & J=L \\
-\frac{3 L-1}{L(2 L-1)} & J=L-1
\end{array}\right\} .
\end{gathered}
$$

Note that $H_{3}=H_{3}^{C}+H_{3}^{B}$ has both a Coulomb term and a Breit term .

In Table 2 the Coulomb energy corrections, $E_{C}=H_{3}^{C}+H_{4}^{C}$, are compared with the Breit energy corrections, $E_{B}=H_{2}^{B}+H_{3}^{B}+H_{5}^{B}$. Also shown in Table 2 are the total Pauli energies $E_{P}^{\prime}-2 m c^{2}$ (49) in Hartree which can be compared to $E_{P}-2 m c^{2}$ (23) in Table 1. This comparison allows one to determine the electric and magnetic contributions to the fine structure. It is important to realize that the Breit energy correction is found from the expectation value of the Breit potential for the various states and not by a diagonalization as was the case for the Coulomb potential. This means that if one adds the energy corrections for the Breit terms and $H_{a n}$ to both $E_{D}$ and $E_{P}$ in Table 1, the differences $E_{D}-E_{P}$ remain the same to order $m c^{2} \alpha^{6}$. Table 1 and Table 2 allow one to understand the two-body Dirac equation in the Coulomb gauge in the context of positronium spectroscopy.

\section{Anomalous States}

The energies of the anomalous bound-states are considerably shifted when the magnetic potential is included in the Hamiltonian. The magnetic part of the potential can be evaluated much more easily for the anomalous states than for the atomic states. However, the Breit 


\begin{tabular}{|c|c|c|c|}
\hline$n L L S$ & $\boldsymbol{E}_{C}\left(10^{-9}\right)$ & $\boldsymbol{E}_{B}\left(10^{-9}\right)$ & $E_{P}^{\prime}-2 m c^{2}$ \\
\hline 1000 & 2497.46923 & 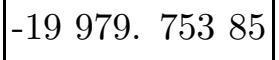 & -0.25001748228462 \\
\hline $\begin{array}{llll}1 & 0 & 1 & 1\end{array}$ & 2497.46923 & -2 219. 97265 & -0.24998640266752 \\
\hline 2000 & 156. 09183 & -2 913. 71410 & -0.06250275762228 \\
\hline 2011 & 156. 09183 & -693.74145 & $-0.062498 \quad 87267014$ \\
\hline 2101 & -121.75404 & -416.24487 & -0.06250053764962 \\
\hline 2110 & -398. 90134 & -1248.73462 & -0.06250164763595 \\
\hline 2111 & -260.15345 & -554.99316 & -0.06250081514621 \\
\hline 2112 & 17. 34354 & -166.49795 & -0.06250014915441 \\
\hline 3000 & 30. 83295 & -904.43330 & -0.02777865137813 \\
\hline 3011 & 30. 83295 & -246.66363 & -0.02777750028120 \\
\hline 3101 & -51.38826 & -164.44242 & -0.02777799360845 \\
\hline 3110 & -133.60947 & -411.10605 & -0.027 77832249329 \\
\hline 3111 & -92.49886 & -205.55302 & -0.02777807582966 \\
\hline 3112 & -10.27765 & -90.44333 & -0.027 77787849876 \\
\hline 3202 & -18. 49977 & $-65.776 \quad 97$ & -0.02777786205452 \\
\hline 3211 & -43.16613 & -123.33181 & -0.02777794427573 \\
\hline 3212 & -26. 72189 & -73.99909 & -0.02777787849876 \\
\hline 3213 & -2.05553 & -35.23766 & -0.02777781507097 \\
\hline
\end{tabular}

TABLE II: Coulomb, Breit, and Total Pauli Energies in Hartree

potential cannot be used for the anomalous bound-states because the magnetic potential is as strong as the Coulomb potential. In fact, for the anomalous state delta functions $\delta\left(\rho-\rho^{\prime}\right)$, which have very high momentum $\hbar k \gg m c$, the Dirac operators $\boldsymbol{\alpha}_{e}$ and $\boldsymbol{\alpha}_{p}$ have expectation values of $v / c=1$ and not $v / c \sim \alpha$ as in the case for atomic states. For such high momentum states, one must now use the covariant Feynman gauge instead of the Coulomb gauge. For this gauge, one uses the Gaunt potential $\boldsymbol{V}_{G}$ [11] instead of the Breit potential $V_{B}$ where

$$
\boldsymbol{V}_{M}(\rho)=\boldsymbol{V}_{G}(\rho)=\frac{e^{2}}{\rho} \boldsymbol{\alpha}_{e} \cdot \boldsymbol{\alpha}_{p}
$$

or, equivalently, 


$$
\boldsymbol{V}_{G}(\rho)=\frac{e^{2}}{\rho}\left(\begin{array}{cccc}
0 & 0 & 0 & \boldsymbol{\sigma}_{e} \cdot \boldsymbol{\sigma}_{p} \\
0 & 0 & \boldsymbol{\sigma}_{e} \cdot \boldsymbol{\sigma}_{p} & 0 \\
0 & \boldsymbol{\sigma}_{e} \cdot \boldsymbol{\sigma}_{p} & 0 & 0 \\
\boldsymbol{\sigma}_{e} \cdot \boldsymbol{\sigma}_{p} & 0 & 0 & 0
\end{array}\right) .
$$

For the singlet and triplet spin functions $\Omega_{0}^{0}$ and $\Omega_{\Sigma}^{1}$, respectively, one finds

$$
\begin{aligned}
& \left(\boldsymbol{\sigma}_{e} \cdot \boldsymbol{\sigma}_{p}\right) \Omega_{0}^{0}=-3 \Omega_{0}^{0}, \\
& \left(\boldsymbol{\sigma}_{e} \cdot \boldsymbol{\sigma}_{p}\right) \Omega_{\Sigma}^{1}=\Omega_{\Sigma}^{1} .
\end{aligned}
$$

such that

$$
\begin{aligned}
& \left(\boldsymbol{\alpha}_{e} \cdot \boldsymbol{\alpha}_{p}\right) \Omega_{0}^{0}\left(\boldsymbol{e}_{11}-\boldsymbol{e}_{22}\right)=3 \Omega_{0}^{0}\left(\boldsymbol{e}_{11}-\boldsymbol{e}_{22}\right) \\
& \left(\boldsymbol{\alpha}_{e} \cdot \boldsymbol{\alpha}_{p}\right) \Omega_{0}^{0}\left(\boldsymbol{e}_{12}-\boldsymbol{e}_{21}\right)=3 \Omega_{0}^{0}\left(\boldsymbol{e}_{12}-\boldsymbol{e}_{21}\right) \\
& \left(\boldsymbol{\alpha}_{e} \cdot \boldsymbol{\alpha}_{p}\right) \Omega_{\Sigma}^{1}\left(\boldsymbol{e}_{11}-\boldsymbol{e}_{22}\right)=-\Omega_{\Sigma}^{1}\left(\boldsymbol{e}_{11}-\boldsymbol{e}_{22}\right) \\
& \left(\boldsymbol{\alpha}_{e} \cdot \boldsymbol{\alpha}_{p}\right) \Omega_{\Sigma}^{1}\left(\boldsymbol{e}_{12}-\boldsymbol{e}_{21}\right)=-\Omega_{\Sigma}^{1}\left(\boldsymbol{e}_{12}-\boldsymbol{e}_{21}\right) .
\end{aligned}
$$

Importantly, the anomalous bound-states are eigenfunctions of the total potentials $V_{C}, V_{G}$, and $E=V_{C}+V_{G}$. From (54) and (41), the eigenvalues of the potentials $V_{C}, V_{G}$, and $E=V_{C}+V_{G}$ are given in (55).

\begin{tabular}{c|c|c|c|c|c|} 
State & \multicolumn{1}{c}{$\psi(\rho)\left[Y^{L} \Omega^{S}\right]^{J}$} & \multicolumn{1}{c}{ Dirac Vector } & $V_{C}$ & $V_{G}$ & $E=V_{C}+V_{G}$ \\
$\left|\Psi_{S}^{0}, i\right\rangle$ & $\sqrt{\Delta \rho} \delta\left(\rho-\rho_{i}\right)\left[Y^{0} \Omega^{0}\right]^{0}$ & $\left(\boldsymbol{e}_{11}-\boldsymbol{e}_{22}\right) / \sqrt{2}$ & $-\frac{e^{2}}{\rho_{i}}$ & $\frac{3 e^{2}}{\rho_{i}}$ & $\frac{2 e^{2}}{\rho_{i}}$ \\
$\left|\Psi_{A}^{0}, i\right\rangle$ & $\sqrt{\Delta \rho} \delta\left(\rho-\rho_{i}\right)\left[Y^{0} \Omega^{0}\right]^{0}$ & $\left(\boldsymbol{e}_{12}-\boldsymbol{e}_{21}\right) / \sqrt{2}$ & $-\frac{e^{2}}{\rho_{i}}$ & $\frac{3 e^{2}}{\rho_{i}}$ & $\frac{2 e^{2}}{\rho_{i}}$ \\
$\left|\Psi_{A}^{\alpha}, i\right\rangle$ & $\sqrt{\Delta \rho} \delta\left(\rho-\rho_{i}\right)\left[Y^{1} \Omega^{1}\right]^{0}$ & $\left(\boldsymbol{e}_{12}-\boldsymbol{e}_{21}\right) / \sqrt{2}$ & $-\frac{e^{2}}{\rho_{i}}$ & $-\frac{e^{2}}{\rho_{i}}$ & $-\frac{2 e^{2}}{\rho_{i}}$ \\
$\left|\Psi_{S}^{\alpha}, i\right\rangle$ & $\sqrt{\Delta \rho} \delta\left(\rho-\rho_{i}\right)\left[Y^{1} \Omega^{1}\right]^{0}$ & $\left(\boldsymbol{e}_{11}-\boldsymbol{e}_{22}\right) / \sqrt{2}$ & $-\frac{e^{2}}{\rho_{i}}$ & $-\frac{e^{2}}{\rho_{i}}$ & $-\frac{2 e^{2}}{\rho_{i}}$ \\
\cline { 2 - 5 }
\end{tabular}

It is interesting that there is a doublet for the anomalous states instead of a singlet or a triplet as in the case of atomic states. In the case of anomalous bound-states, the effective mass $m c^{2}= \pm 2 e^{2} / \rho_{i}$ depends on the spin components and the degeneracy depends on the Dirac components. This is the reverse of the atomic case. One can also view the doublets $\left|\Psi_{A}^{\alpha}, i\right\rangle$ and $\left|\Psi_{S}^{\alpha}, i\right\rangle$ to be the antiparticles of the doublets $\left|\Psi_{S}^{0}, i\right\rangle$ and $\left|\Psi_{A}^{0}, i\right\rangle$ because they have the opposite mass. Furthermore, the delta functions $\delta\left(\rho-\rho_{i}\right)$ for the anomalous boundstates $|\Psi, i\rangle$ show that the electron and positron cannot overlap and annihilate. The lack of overlap between $\delta\left(\rho-\rho_{i}\right)$ and $\delta\left(\rho-\rho_{j}\right)$ also means that there can be no radiative transitions 
between anomalous states $|\Psi, i\rangle$ and $|\Psi, j\rangle$. That is, for $\rho_{i} \neq \rho_{j}$, there are no multipole moments between anomalous bound-states $|\Psi, i\rangle$ and $|\Psi, j\rangle$ where

$$
\left\langle\delta\left(\rho-\rho_{i}\right)\left|\rho^{n}\right| \delta\left(\rho-\rho_{j}\right)\right\rangle=0
$$

\section{BETHE-SALPETER EQUATIONS FOR POSITRONIUM: SEPARABILITY OF ATOMIC AND ANOMALOUS STATES}

It is now shown that the Bethe-Salpeter equation insures the complete separability between the atomic and anomalous states. That is, in the presence of potentials $V_{C}$ and $V_{M}$ above, the atomic and anomalous states cannot interact. Although we treat only $V_{C}$ here, the same result applies to $V_{M}$. This means that the Dirac wave functions in Fig. 1 and Fig. 5 are incorrect because the Pauli and anomalous wave functions are erroneously coupled by the Coulomb potential $V_{C}$ in the two-body Dirac equation. Only the Pauli wave functions in Fig. 1 are correct near the origin for the order calculated.

The two-body Dirac equation for positronium is only an approximation to the BetheSalpeter equation [3] which is relativistically invariant and can include all the necessary QED (quantum electrodynamic) corrections. Ultimately, any justification for using the twobody Dirac equation for positronium comes from the Bethe-Salpeter equation. Furthermore, the Bethe-Salpeter equation itself has been related to S-matrix field theory by Gell-Mann and Low [12] and Sucher [13] to justify its application to bound-states. Thus, the Bethe-Salpeter equation may be thought of as equivalent to bound-state QED, whereas the original S-matrix field theory was applied to scattering QED. More recent treatments of bound-state QED include works by Sapirstein and Yennie [14], Ito [15], and Grant [16]. However, one must keep in mind that the anomalous bound-states can only occur for equal mass atoms such as positronium and not for the general hydrogenic atom originally considered by Salpeter and others. In this section, natural units are used where $c=\hbar=1$.

It is useful to define the times $\tau$ and $T$ as the fourth components of the relative coordinate four vectors $\rho \equiv(\boldsymbol{\rho}, i \tau)$ and $R \equiv(\boldsymbol{R}, i T)$, respectively in (5). One can also define the energies $\varepsilon$ and $E$ as the fourth components of the conjugate momentum four vectors $\pi \equiv(\boldsymbol{\pi}, i \varepsilon)$ and $P \equiv(\boldsymbol{P}, i E)$, respectively in ([6) . Note that the notation is changed in this section so that $\rho$, for example, is now a four-vector and is not equal to $|\boldsymbol{\rho}|$ which will now be written out explicitly. From (5) one finds the times 


$$
\tau=t_{e}-t_{p}, \quad T=\frac{1}{2}\left(t_{e}+t_{p}\right)
$$

and from (66) the conjugate energies

$$
\varepsilon=\frac{1}{2}\left(e_{e}-e_{p}\right), \quad E=e_{e}+e_{p},
$$

in terms of the one-body times and energies. The problem with the two-body Dirac equation is that its Hamiltonian formalism does not properly treat the relative time $\tau$ in the advanced or retarded potential nor the relative energy $\varepsilon$. Indeed, the two-body Dirac equation is only a function of the time $T$ and energy $E$. Thus, it is implicit that $\tau=0$ so that $t_{e}=t_{p}=T$ and $\varepsilon=0$ so that $e_{e}=e_{p}=E / 2$. It has already been shown that the anomalous states of the two-body Dirac equation are coupled to the atomic states by the Coulomb potential. This coupling is responsible for the fact that $y_{11}^{2}=y_{22}^{2}$ near the origin in Fig. 5 . The Bethe-Salpeter equation is now used for a Coulomb potential, treating the relative time and energy $\tau$ and $\varepsilon$ explicitly, in order to show that these anomalous solutions of positronium are actually uncoupled from the atomic solutions.

The Feynman derivation of QED for positronium [17] is based on the one-body Green's function for the one-body Dirac equation. Similarly the Bethe-Salpeter equation is based on the two-body Green's function for the two-body Dirac equation. The two-body Dirac equation for positronium with a Coulomb potential is only accurate to order $m \alpha^{4}$ [18]. Indeed Karplus and Klein [19] and Fulton and Martin [9] have given the corrections to order $m \alpha^{5}$ for positronium using the Bethe-Salpeter equation [3], [18]. This equation is now used to derive the atomic and anomalous solutions to positronium in a Coulomb potential, which differ significantly from those of the two-body Dirac equation.

The two-body Green's function for the electron and positron is simply the product of the one-body Green's functions $K\left(r_{e}, r_{e}^{\prime}\right) K\left(r_{p}, r_{p}^{\prime}\right) \equiv K^{2}$ and is a solution of the two-body equation,

$$
\left[i\left(\gamma^{e} \cdot p_{e}\right)+m_{e}\right]\left[i\left(\gamma^{p} \cdot p_{p}\right)+m_{p}\right] K^{2}=\delta^{4}\left(r_{e}-r_{e}^{\prime}\right) \delta^{4}\left(r_{p}-r_{p}^{\prime}\right)
$$

without stipulating the boundary conditions. Transforming to the relative coordinates in the momentum representation with $\boldsymbol{P}=\mathbf{0}$ where $\boldsymbol{\pi}=\boldsymbol{p}_{e}=-\boldsymbol{p}_{p}$ and using $\boldsymbol{\alpha}=i \beta \boldsymbol{\gamma}$, 
$\beta=\gamma_{4}$ one finds

$$
K^{2}=-\frac{\gamma_{4}^{e}}{\left[\varepsilon+\frac{1}{2} E-\boldsymbol{h}_{0}^{e}\right]} \frac{\gamma_{4}^{p}}{\left[\varepsilon-\frac{1}{2} E+\boldsymbol{h}_{0}^{p}\right]}
$$

where

$$
\begin{aligned}
\boldsymbol{h}_{0}^{e} \psi_{ \pm}^{e}(\boldsymbol{\pi}) & = \pm e_{0} \psi_{ \pm}^{e}(\boldsymbol{\pi}), \\
\boldsymbol{h}_{0}^{p} \psi_{ \pm}^{p}(-\boldsymbol{\pi}) & = \pm e_{0} \psi_{ \pm}^{p}(-\boldsymbol{\pi}) \\
e_{0} & =\sqrt{\pi^{2}+m^{2}} .
\end{aligned}
$$

The Coulomb potential $V_{C}(\rho)$ in the momentum representation is

$$
G_{C}(-\boldsymbol{k})=-\frac{1}{(2 \pi)^{3}} \int d^{3} \rho e^{i \boldsymbol{k} \cdot \rho} \frac{e^{2}}{\rho}=-\frac{e^{2}}{2 \pi^{2}} \frac{1}{\boldsymbol{k} \cdot \boldsymbol{k}} .
$$

The two-body Bethe-Salpeter equation in the momentum representation for the Coulomb potential becomes

$$
\begin{aligned}
\Psi(\boldsymbol{\pi}) & =-\frac{\gamma_{4}^{e} \gamma_{4}^{p}}{2 \pi i} \int d^{3} k \int_{-\infty}^{\infty} d \varepsilon K^{2} G_{C}(-\boldsymbol{k}) \Psi(\boldsymbol{k}+\boldsymbol{\pi}), \\
& =\frac{1}{2 \pi i} \int d^{3} k \int_{-\infty}^{\infty} d \varepsilon \frac{1}{\left[\varepsilon+\frac{1}{2} E-\boldsymbol{h}_{0}^{e}\right]} \frac{1}{\left[\varepsilon-\frac{1}{2} E+\boldsymbol{h}_{0}^{p}\right]} G_{C}(-\boldsymbol{k}) \Psi(\boldsymbol{k}+\boldsymbol{\pi}),
\end{aligned}
$$

where it is assumed, for simplicity, that $\Psi$ is independent of energy $\varepsilon$ although an equivalent equation occurs under more general conditions [18]. Projecting with operators $\boldsymbol{\Lambda}_{ \pm \pm}$on (60), where

$$
\begin{aligned}
\boldsymbol{\Lambda}_{ \pm \pm} & =\boldsymbol{\Lambda}_{ \pm}^{e}(\boldsymbol{\pi}) \boldsymbol{\Lambda}_{ \pm}^{p}(-\boldsymbol{\pi})=\left|\psi_{ \pm}^{e}(\boldsymbol{\pi}) \psi_{ \pm}^{p}(-\boldsymbol{\pi})\right\rangle\left\langle\psi_{ \pm}^{e}(\boldsymbol{\pi}) \psi_{ \pm}^{p}(-\boldsymbol{\pi})\right| \\
& =\frac{e_{0} \pm \boldsymbol{h}_{0}^{e}(\boldsymbol{\pi})}{2 e_{0}} \frac{e_{0} \pm \boldsymbol{h}_{0}^{p}(\boldsymbol{\pi})}{2 e_{0}}
\end{aligned}
$$

one finds

$$
\begin{aligned}
\Psi_{ \pm \pm}(\boldsymbol{\pi}) & =\frac{\mathbf{1}}{2 \pi i} \int_{-\infty}^{\infty} d \varepsilon \frac{1}{\left[\varepsilon-\varepsilon_{e}\right]} \frac{1}{\left[\varepsilon-\varepsilon_{p}\right]} \Gamma_{ \pm \pm}(\boldsymbol{\pi}) \\
\Gamma_{ \pm \pm}(\boldsymbol{\pi}) & =\boldsymbol{\Lambda}_{ \pm \pm} \int d^{3} k G(-\boldsymbol{k}) \Psi(\boldsymbol{k}+\boldsymbol{\pi})
\end{aligned}
$$

where $K^{2}$ has poles at

$$
\varepsilon_{e}=-\frac{1}{2} E \pm e_{0}, \quad \varepsilon_{p}=\frac{1}{2} E \mp e_{0},
$$

corresponding to the first and second terms in the denominator of (59), respectively, when operating on $\psi_{ \pm}^{e}(\boldsymbol{\pi}) \psi_{ \pm}^{p}(-\boldsymbol{\pi})$. The wave functions $\Psi_{ \pm \pm}(\boldsymbol{\pi})$ are now evaluated below where it 
is shown that one must use the Feynman propagator $K=K_{F}$ for all atomic states and the Retarded propagator $K=K_{R}$ for anomalous bound-states.

For both $K_{F}$ and $K_{R}$ one chooses $e=e_{0}-i \epsilon$ so that positive-energy states $\psi_{+}$propagate forward in time. The question becomes: how should negative-energy states $\psi_{\text {- propagate }}$ in time? For the Feynman single particle propagator $K_{F}$ one chooses $e=-e_{0}+i \epsilon$ so that

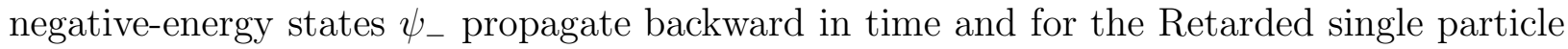
propagator $K_{R}$ one chooses $e=-e_{0}-i \epsilon$ so that negative-energy states $\psi_{\text {- propagate forward }}$ in time. So one has the following results for the time propagation of positive- and negativeenergy states:

$$
\begin{aligned}
& K_{F}\left\{\begin{array}{cc}
\psi_{+}: e=+e_{0}-i \epsilon, & \text { forward } \\
\psi_{-}: e=-e_{0}+i \epsilon, & \text { backward }
\end{array}\right\} \text { all atomic states, } \\
& K_{R}\left\{\begin{array}{ll}
\psi_{+}: e=+e_{0}-i \epsilon, & \text { forward } \\
\psi_{-}: e=-e_{0}-i \epsilon, & \text { forward }
\end{array}\right\} \text { anomalous bound-states. }
\end{aligned}
$$

The correct temporal boundary condition on the negative-energy states $\psi_{\text {- depends on }}$ physical consistency with the known temporal behavior of the electron or positron undergoing scattering or annihilation. For free particles, both negative-energy electrons and positrons must propagate backward in time for two important reasons [5, 17]. First, if a negative-energy particle propagated forward in time, then a positive-energy particle could scatter into a negative-energy particle and be lost at a later time. This is not possible because of particle conservation. Second, a virtual negative-energy electron must propagate backward in time so that it can annihilate with a virtual positive-energy electron which is moving forward in time. That is, a virtual negative-energy electron moving backward in time is equivalent to a positron moving forward in time. Such time behavior accounts for virtual electron-positron pairs. This boundary condition $e=-e_{0}+i \epsilon$ for negative-energy states $\psi_{-}$of free particles is determined from physical reasoning although both boundary conditions are mathematically allowed. Thus, for $K$ to correspond to propagation of free electrons or positrons, one must choose $e= \pm e_{0} \mp i \epsilon$ for the proper boundary conditions corresponding to $K_{F}$. We show below that this choice of $K_{F}$ also must also apply to atomic bound-states. However, it will also be shown below that $K_{F}$ cannot be used for electrons or positrons in anomalous bound-states where the particles are not free. 


\section{Atomic State Propagator $K_{F}^{2}$}

One can now use the temporal boundary conditions for $K=K_{F}$ in (62). Consider first the poles of $\Psi_{++}(\boldsymbol{\pi})$ in (61) at

$$
\varepsilon_{e}=-\frac{1}{2} E+e_{0}-i \epsilon, \quad \varepsilon_{p}=\frac{1}{2} E-e_{0}+i \epsilon
$$

One can complete the line integral in either the upper or lower half complex plane where the integrand is convergent with the same results

$$
\begin{aligned}
\Psi_{++}(\boldsymbol{\pi}) & =\frac{1}{2 \pi i} \int_{-\infty}^{\infty} d \varepsilon \frac{1}{\left[\varepsilon-\varepsilon_{e}\right]\left[\varepsilon-\varepsilon_{p}\right]} \Gamma_{++}(\boldsymbol{\pi}), \\
& =\frac{1}{E-2 e_{0}} \Gamma_{++}(\boldsymbol{\pi}) .
\end{aligned}
$$

Similarly, for the poles of $\Psi_{--}(\boldsymbol{\pi})$ in (61) at

$$
\varepsilon_{e}=-\frac{1}{2} E-e_{0}+i \epsilon, \quad \varepsilon_{p}=\frac{1}{2} E+e_{0}-i \epsilon,
$$

the integral becomes

$$
\begin{aligned}
\Psi_{--}(\boldsymbol{\pi}) & =\frac{1}{2 \pi i} \int_{-\infty}^{\infty} d \varepsilon \frac{1}{\left[\varepsilon-\varepsilon_{e}\right]\left[\varepsilon-\varepsilon_{p}\right]} \Gamma_{--}(\boldsymbol{\pi}) \\
& =-\frac{1}{E+2 e_{0}} \Gamma_{--}(\boldsymbol{\pi})
\end{aligned}
$$

Finally, for the $K_{F}$ propagator, one finds for the corresponding integrals in (61) that $\Psi_{+-}(\boldsymbol{\pi})=\Psi_{-+}(\boldsymbol{\pi})=0$. Combining these results and letting

$$
\Lambda=\Lambda_{++}(\boldsymbol{\pi})-\Lambda_{--}(\boldsymbol{\pi}),
$$

one has

$$
\left(E-2 e_{0}\right) \Psi_{++}(\boldsymbol{\pi})+\left(E+2 e_{0}\right) \Psi_{--}(\boldsymbol{\pi})=\boldsymbol{\Lambda} \Gamma(\boldsymbol{\pi}),
$$

or

$$
\left[\boldsymbol{h}_{0}^{e}(\boldsymbol{\pi})+\boldsymbol{h}_{0}^{p}(\boldsymbol{\pi})\right]\left[\Psi_{++}(\boldsymbol{\pi})+\Psi_{--}(\boldsymbol{\pi})\right]-\frac{e^{2}}{2 \pi^{2}} \boldsymbol{\Lambda} \int d^{3} k \frac{1}{\boldsymbol{k} \cdot \boldsymbol{k}} \Psi(\boldsymbol{k}+\boldsymbol{\pi})=E\left[\Psi_{++}(\boldsymbol{\pi})+\Psi_{--}(\boldsymbol{\pi})\right],
$$

$$
\Psi_{+-}(\boldsymbol{\pi})=\Psi_{-+}(\boldsymbol{\pi})=0
$$

which is the Bethe-Salpeter equation for the atomic states for a Coulomb potential in the ladder approximation. 
Because of the operator $\Lambda$, the Coulomb potential is attractive for positive-energy atomic states but is repulsive for negative-energy atomic states. Thus the positive and negative wave functions have bound-states with the opposite energies. Only the free particle states $\Psi_{++}(\boldsymbol{\pi})$ and $\Psi_{--}(\boldsymbol{\pi})$ with energies $E=2 e_{0}$ and $E=-2 e_{0}$, respectively, contribute to the atomic states for a Coulomb potential to all orders in the ladder approximation.

One can compare the above equation to the two-body Dirac equation in the momentum representation

$$
\left[\boldsymbol{h}_{0}^{e}(\boldsymbol{\pi})+\boldsymbol{h}_{0}^{p}(\boldsymbol{\pi})\right] \Psi(\boldsymbol{\pi})-\frac{e^{2}}{2 \pi^{2}} \int d^{3} k \frac{1}{\boldsymbol{k} \cdot \boldsymbol{k}} \Psi(\boldsymbol{k}+\boldsymbol{\pi})=E \Psi(\boldsymbol{\pi}),
$$

which erroneously includes the anomalous states $\Psi_{+-}(\boldsymbol{\pi})$ and $\Psi_{-+}(\boldsymbol{\pi})$. The solutions to (64) in the coordinate representation lead to the Dirac solutions shown in Fig. 1 while the solutions to (63) lead to the Pauli solutions shown in Fig. 1 (to the calculated order). The Dirac solutions in Fig. 1 and Fig. 5 are incorrect because they include Coulomb coupling between the atomic and anomalous bound-states which cannot occur.

\section{Anomalous State Propagator $K_{R}^{2}$}

The anomalous bound-states which include the $\Psi_{+_{-}}(\boldsymbol{\pi})$ and $\Psi_{-+}(\boldsymbol{\pi})$ wave functions are indeed solutions of the Bethe-Salpeter equation if one changes the boundary conditions. The appropriate boundary conditions for anomalous states correspond to both positiveand negative-energy states propagating forward in time $\tau$ corresponding to the two-body Retarded propagator $K_{R}^{2}$. That is, one can now use the temporal boundary conditions for $K=K_{R}$ in (62). This means that the negative-energy states which comprise the anomalous bound-states cannot exist as separate free particles: negative-energy states of free particles must propagate backward in time because they correspond to the antiparticle propagating forward in time.

But this is not a problem for anomalous bound-states. Probability is still conserved for bound-states despite the fact that negative- and positive-energy states can scatter into each other. Further, there can be no electron-positron annihilation for such states as shown in Sec. III. This means that the discrete variable representation (DVR) is necessary to produce the correct boundary conditions for the proper $K_{R}^{2}$ propagation. It appears that anomalous bound-states are both mathematically and physically allowed. 
With this proper time behavior, the poles of $\Psi_{+-}(\boldsymbol{\pi})$ in (61) are

$$
\varepsilon_{e}=-\frac{1}{2} E+e_{0}-i \epsilon, \quad \varepsilon_{p}=\frac{1}{2} E+e_{0}+i \epsilon
$$

The integral for the Bethe-Salpeter equation becomes

$$
\Psi_{+-}(\boldsymbol{\pi})=\frac{1}{E} \Gamma_{+-}(\boldsymbol{\pi}),
$$

where one may close the line integral in either the upper- or lower-half complex plane. Similarly, the poles of $\Psi_{-+}(\boldsymbol{\pi})$ in (61) are

$$
\varepsilon_{e}=-\frac{1}{2} E-e_{0}-i \epsilon, \quad \varepsilon_{p}=\frac{1}{2} E-e_{0}+i \epsilon,
$$

and the integral becomes

$$
\Psi_{-+}(\boldsymbol{\pi})=\frac{1}{E} \Gamma_{-+}(\boldsymbol{\pi}) .
$$

Finally, for the $K_{R}$ propagator, one finds for the corresponding integrals in (61) that $\Psi_{++}(\boldsymbol{\pi})=\Psi_{--}(\boldsymbol{\pi})=0$. Combining these results, one has

$$
\begin{aligned}
-\frac{e^{2}}{2 \pi^{2}}\left[\boldsymbol{\Lambda}_{+-}(\boldsymbol{\pi})+\Lambda_{-+}(\boldsymbol{\pi})\right] \int d^{3} k \frac{1}{\boldsymbol{k} \cdot \boldsymbol{k}} \Psi(\boldsymbol{k}+\boldsymbol{\pi}) & =E\left[\Psi_{+-}(\boldsymbol{\pi})+\Psi_{-+}(\boldsymbol{\pi})\right], \\
\Psi_{++}(\boldsymbol{\pi}) & =\Psi_{--}(\boldsymbol{\pi})=0,
\end{aligned}
$$

which is the Bethe-Salpeter equation for the anomalous bound-states for a Coulomb potential in the ladder approximation. The solutions to this equation lead to the anomalous bound-state energies shown in Fig. 2 and wave functions shown in Figs. 3 and 4. As a result, the anomalous bound-states formed from $\Psi_{+-}(\boldsymbol{\pi})$ and $\Psi_{-+}(\boldsymbol{\pi})$ arise from the ladder terms of QED and never couple with atomic states. Finally, note that, for atomic and anomalous bound-states, the Bethe-Salpeter equation leaves no choice in the temporal boundary conditions but rather they are determined automatically by this equations.

\section{CONCLUSIONS}

It has been shown that there are two types of bound-state solutions to the two-body Dirac equation and Bethe-Salpeter equation for positronium: there are the normal atomic solutions and the anomalous solutions. The energies and wave functions for these two solution have been derived by solving both the two-body Dirac equation and the Bethe-Salpeter equation 
with an electromagnetic potential. The anomalous bound-states wave functions are Dirac delta functions in the radial coordinate corresponding to the discrete variable representation (DVR). For these highly localized wave functions, the electron and positron can neither radiate nor annihilate.

It has also been shown that the numerical accuracy of the atomic Dirac energies are of order $m c^{2} \alpha^{6}$ or less when compared to the analytic Pauli energies. The Dirac boundstate wave function radial components, however, differs significantly near the origin from their Pauli approximations and are incorrect. This difference is because the Dirac equation erroneously couples the atomic ground-state wave function with the anomalous bound-state wave functions near the origin due to the electromagnetic potential. No such coupling can occurs for the Bethe-Salpeter equation because of the different time behavior for the atomic and anomalous negative-energy states.

Finally, it has been shown that one must use the Feynman two-body propagator $K_{F}^{2}$ for the atomic bound-states. On the other hand, one must use the Retarded two-body propagator $K_{R}^{2}$ for the anomalous bound-states. Unlike atomic states, anomalous states can never be free. For the atomic bound-states, the free particle states are useful where the momentum is quantized. On the other hand, for the anomalous bound-states, the discrete variable representation (DVR) must be used where the position is quantized.

\section{Appendix A: Coordinate Representation for Two-Body Dirac Equations}

These equations are given for a free particle in spherical coordinates. For a Coulomb potential replace the energy $E$ by $E-V_{C}$ below where $V_{C}=-e^{2} / \rho$. We define the recoupling coefficients $a$ and $b$ as in (16). The three cases 1A, 2A, and 3A of equations below are in

agreement with those of Malenfant (see Refs. [4] and [1]) for sets 1, 3, and 2, respectively using the explicitly symmetrized basis for the wave function coefficients. Note that the radial functions for all cases, $y_{i j}(\rho)$, include the radial scale factor $\rho$ for which one has the boundary condition $y_{i j}(0)=0$. The three different cases below are labeled by their dominant $\Psi_{11}$ component for the atomic states. 
1. Case 1A: $S=0, L=J$.

For the basis,

$$
\Psi=\frac{1}{\rho}\left(\begin{array}{c}
y_{11}^{0}(\rho)\left[Y^{J} \Omega^{0}\right]_{N}^{J} \\
i\left\{y_{12}^{+}(\rho)\left[Y^{J+1} \Omega^{1}\right]_{N}^{J}+y_{12}^{-}(\rho)\left[Y^{J-1} \Omega^{1}\right]_{N}^{J}\right\} \\
i\left\{y_{21}^{+}(\rho)\left[Y^{J+1} \Omega^{1}\right]_{N}^{J}+y_{21}^{-}(\rho)\left[Y^{J-1} \Omega^{1}\right]_{N}^{J}\right\} \\
y_{22}^{0}(\rho)\left[Y^{J} \Omega^{0}\right]_{N}^{J}
\end{array}\right)
$$

one has, using (15)-(18),

$$
\begin{aligned}
2 m c^{2}\left(y_{11}^{0}-y_{22}^{0}\right)-2 a \hbar c\left[\frac{d}{d \rho}+\frac{J+1}{\rho}\right]\left(y_{12}^{+}+y_{21}^{+}\right)+2 b \hbar c\left[\frac{d}{d \rho}-\frac{J}{\rho}\right]\left(y_{12}^{-}+y_{21}^{-}\right) & =E\left(y_{11}^{0}+y_{22}^{0}\right), \\
2 m c^{2}\left(y_{11}^{0}+y_{22}^{0}\right) & =E\left(y_{11}^{0}-y_{22}^{0}\right), \\
2 a \hbar c\left[\frac{d}{d \rho}-\frac{J+1}{\rho}\right]\left(y_{11}^{0}+y_{22}^{0}\right) & =E\left(y_{12}^{+}+y_{21}^{+}\right), \\
-2 b \hbar c\left[\frac{d}{d \rho}+\frac{J}{\rho}\right]\left(y_{11}^{0}+y_{22}^{0}\right) & =E\left(y_{12}^{-}+y_{21}^{-}\right), \\
0 & =E\left(y_{12}^{+}-y_{21}^{+}\right), \\
0 & =E\left(y_{12}^{-}-y_{21}^{-}\right) .
\end{aligned}
$$

The last two equations above are mathematically allowed but have the opposite chargeconjugation parity from the first four coupled equations. As a result, these last two equations are uncoupled from the first four equations.

2. Case 2A: $S=1, L=J$.

For the basis

$$
\Psi=\frac{1}{\rho}\left(\begin{array}{c}
y_{11}^{1}(\rho)\left[Y^{J} \Omega^{1}\right]_{N}^{J} \\
i\left\{y_{12}^{+}(\rho)\left[Y^{J+1} \Omega^{1}\right]_{N}^{J}+y_{12}^{-}(\rho)\left[Y^{J-1} \Omega^{1}\right]_{N}^{J}\right\} \\
i\left\{y_{21}^{+}(\rho)\left[Y^{J+1} \Omega^{1}\right]_{N}^{J}+y_{21}^{-}(\rho)\left[Y^{J-1} \Omega^{1}\right]_{N}^{J}\right\} \\
y_{22}^{1}(\rho)\left[Y^{J} \Omega^{1}\right]_{N}^{J}
\end{array}\right)
$$


one has the equations

$$
\begin{aligned}
2 m c^{2}\left(y_{11}^{1}+y_{22}^{1}\right)+2 b \hbar c\left[\frac{d}{d \rho}+\frac{J+1}{\rho}\right]\left(y_{12}^{+}-y_{21}^{+}\right)+2 a \hbar c\left[\frac{d}{d \rho}-\frac{J}{\rho}\right]\left(y_{12}^{-}-y_{21}^{-}\right) & =E\left(y_{11}^{1}-y_{22}^{1}\right), \\
2 m c^{2}\left(y_{11}^{1}-y_{22}^{1}\right) & =E\left(y_{11}^{1}+y_{22}^{1}\right), \\
-2 b \hbar c\left[\frac{d}{d \rho}-\frac{J+1}{\rho}\right]\left(y_{11}^{1}+y_{22}^{1}\right) & =E\left(y_{12}^{+}-y_{21}^{+}\right), \\
-2 a \hbar c\left[\frac{d}{d \rho}+\frac{J}{\rho}\right]\left(y_{11}^{1}+y_{22}^{1}\right) & =E\left(y_{12}^{-}-y_{21}^{-}\right), \\
0 & =E\left(y_{12}^{+}+y_{21}^{+}\right), \\
0 & =E\left(y_{12}^{-}+y_{21}^{-}\right) .
\end{aligned}
$$

3. Case 3A: $S=1, L \neq J$.

Case 3A can be obtained from Case $1 \mathrm{~A}$ and Case 2A by a simple transformation by using the wave function

$$
\Psi=\frac{1}{\rho}\left(\begin{array}{c}
i\left\{y_{11}^{+}(\rho)\left[Y^{J+1} \Omega^{1}\right]_{N}^{J}+y_{11}^{-}(\rho)\left[Y^{J-1} \Omega^{1}\right]_{N}^{J}\right\} \\
y_{12}^{0}(\rho)\left[Y^{J} \Omega^{0}\right]_{N}^{J}+y_{12}^{1}(\rho)\left[Y^{J} \Omega^{1}\right]_{N}^{J} \\
y_{21}^{0}(\rho)\left[Y^{J} \Omega^{0}\right]_{N}^{J}+y_{21}^{1}(\rho)\left[Y^{J} \Omega^{1}\right]_{N}^{J} \\
i\left\{y_{22}^{+}(\rho)\left[Y^{J+1} \Omega^{1}\right]_{N}^{J}+y_{22}^{-}(\rho)\left[Y^{J-1} \Omega^{1}\right]_{N}^{J}\right\}
\end{array}\right) .
$$

Note that the large-large component $\Psi_{11}$ can now correspond to the atomic state with either $L=J+1$ or $L=J-1$. The new equations can be found from Case 1 A and Case $2 \mathrm{~A}$ by the exchange $m_{e} \leftrightarrow-m_{e}, y_{11} \leftrightarrow y_{21}, y_{22} \leftrightarrow y_{12}$, or, equivalently, the exchange $m_{p} \leftrightarrow-m_{p}$, $y_{11} \leftrightarrow y_{12}, y_{22} \leftrightarrow y_{21}$. The two-body Dirac equation in this basis gives the eight equations 
for the radial functions (of which six are coupled),

$$
\begin{aligned}
-2 a \hbar c\left[\frac{d}{d \rho}+\frac{J+1}{\rho}\right]\left(y_{11}^{+}+y_{22}^{+}\right)+2 b \hbar c\left[\frac{d}{d \rho}-\frac{J}{\rho}\right]\left(y_{11}^{-}+y_{22}^{-}\right) & =E\left(y_{12}^{0}+y_{21}^{0}\right), \\
2 m c^{2}\left(y_{11}^{+}-y_{22}^{+}\right)+2 a \hbar c\left[\frac{d}{d \rho}-\frac{J+1}{\rho}\right]\left(y_{12}^{0}+y_{21}^{0}\right) & =E\left(y_{11}^{+}+y_{22}^{+}\right), \\
2 m c^{2}\left(y_{11}^{-}-y_{22}^{-}\right)-2 b \hbar c\left[\frac{d}{d \rho}+\frac{J}{\rho}\right]\left(y_{12}^{0}+y_{21}^{0}\right) & =E\left(y_{11}^{-}+y_{11}^{-}\right), \\
0 & =E\left(y_{12}^{0}-y_{21}^{0}\right), \\
2 b \hbar c\left[\frac{d}{d \rho}+\frac{J+1}{\rho}\right]\left(y_{11}^{+}-y_{22}^{+}\right)+2 a \hbar c\left[\frac{d}{d \rho}-\frac{J}{\rho}\right]\left(y_{11}^{-}-y_{22}^{-}\right) & =E\left(y_{12}^{1}-y_{21}^{1}\right), \\
2 m c^{2}\left(y_{11}^{+}+y_{22}^{+}\right)-2 b \hbar c\left[\frac{d}{d \rho}-\frac{J+1}{\rho}\right]\left(y_{12}^{1}-y_{21}^{1}\right) & =E\left(y_{11}^{+}-y_{22}^{+}\right), \\
2 m c^{2}\left(y_{11}^{-}+y_{22}^{-}\right)-2 a \hbar c\left[\frac{d}{d \rho}+\frac{J}{\rho}\right]\left(y_{12}^{1}-y_{21}^{1}\right) & =E\left(y_{11}^{-}-y_{11}^{-}\right), \\
0 & =E\left(y_{12}^{1}+y_{21}^{1}\right) .
\end{aligned}
$$

With this transformation, the two separate sets of three coupled equations in the Case 1A and Case 2A basis now become six coupled equations in Case 3. The two uncoupled equations are allowed but have opposite charge-conjugation parity from the other equations. The charge-conjugation and inversion parity is shown clearly in Appendix B.

\section{Appendix B: Momentum Representation for Two-Body Dirac Equations}

Refer to (20), (21) for the definitions of the states $|L, S, k\rangle$ given below. As in the case of the coordinate representation, there are three Cases $1 \mathrm{~B}, 2 \mathrm{~B}$, and $3 \mathrm{~B}$ of equations in the momentum representation. This representation is given for the free particle basis in spherical coordinates. Below, the energies $E_{ \pm \pm}$for the wave functions $\Psi_{ \pm \pm}$correspond to

$$
\begin{aligned}
& E_{++}=+2 e, E_{--}=-2 e, \\
& E_{+-}=E_{-+}=0,
\end{aligned}
$$

where

$$
e=\sqrt{(\hbar c k)^{2}+\left(m c^{2}\right)^{2}}
$$


as in (8) and (91). The anomalous wave functions $\Psi_{+-}$and $\Psi_{-+}$for energies $E_{+-}$and $E_{-+}$ are symmetrized such that

$$
\begin{aligned}
& \Psi_{S}=\left(\Psi_{+-}+\Psi_{-+}\right) / \sqrt{2}, \\
& \Psi_{A}=\left(\Psi_{+-}-\Psi_{-+}\right) / \sqrt{2} .
\end{aligned}
$$

The equations for the free particles are diagonal in $k$. For a Coulomb potential one must include the relevant potential matrices of the spherical Bessel functions for a given $J$ :

$$
\begin{aligned}
& V_{k k^{\prime}}^{0}=\left\langle J, 0, k\left|V_{C}\right| J, 0, k^{\prime}\right\rangle=-N_{J k} N_{J k^{\prime}} \int_{0}^{\rho_{0}} d \rho \rho j_{J}(k \rho) j_{J}\left(k^{\prime} \rho\right), \\
& V_{k k^{\prime}}^{1}=\left\langle J, 1, k\left|V_{C}\right| J, 1, k^{\prime}\right\rangle=V_{k k^{\prime}}^{0}, \\
& V_{k k^{\prime}}^{+}=\left\langle J+1,1, k\left|V_{C}\right| J+1,1, k^{\prime}\right\rangle=-N_{J k} N_{J k^{\prime}} \int_{0}^{\rho_{0}} d \rho \rho j_{J+1}(k \rho) j_{J+1}\left(k^{\prime} \rho\right), \\
& V_{k k^{\prime}}^{-}=\left\langle J-1,1, k\left|V_{C}\right| J-1,1, k^{\prime}\right\rangle=-N_{J k} N_{J k^{\prime}} \int_{0}^{\rho_{0}} d \rho \rho j_{J-1}(k \rho) j_{J-1}\left(k^{\prime} \rho\right), \\
& V_{k k^{\prime}}^{\alpha}=a V_{k k^{\prime}}^{+}+b V_{k k^{\prime}}^{-}, \\
& V_{k k^{\prime}}^{\beta}=-b V_{k k^{\prime}}^{+}+a V_{k k^{\prime}}^{-} .
\end{aligned}
$$

The energies can then be found by replacing $E \rightarrow E \delta_{k k^{\prime}}-V_{k k^{\prime}}^{i}$ with the appropriate $i$ and diagonalizing the resulting matrix for $E$.

The charge-conjugation $C$ parity and inversion $P$ parity of the $\Psi_{++}, \Psi_{--}, \Psi_{S}, \Psi_{A}$ states are given below. The $C$ and $P$ parities of the atomic states $\Psi_{++}, \Psi_{--}$are identical to those given by Malenfant [4] who did not treat the $\Psi_{S}, \Psi_{A}$ anomalous states. For a given case, only those states with the same charge-conjugation parity $C$ and $P$ can be coupled by the Coulomb potential. As in the coordinate representation, the three different cases below are labeled by their dominant $\Psi_{11}$ component for the atomic states.

1. Case 1B: $S=0, L=J$

Letting

$$
\Psi=\frac{1}{\rho}\left(\begin{array}{c}
c_{11}^{0}|J, 0, k\rangle \\
c_{12}^{\alpha}|J \alpha, 1, k\rangle \\
c_{21}^{\alpha}|J \alpha, 1, k\rangle \\
c_{22}^{0}|J, 0, k\rangle
\end{array}\right)
$$


the two-body Dirac equation in the momentum basis, for a given $k, J$, gives the following three coupled equations for the symmetrized Bessel coefficients,

$$
\begin{aligned}
2 m c^{2}\left(c_{11}^{0}-c_{22}^{0}\right)-2 \hbar c k\left(c_{12}^{\alpha}+c_{21}^{\alpha}\right) & =E\left(c_{11}^{0}+c_{22}^{0}\right), \\
2 m c^{2}\left(c_{11}^{0}+c_{22}^{0}\right) & =E\left(c_{11}^{0}-c_{22}^{0}\right), \\
-2 \hbar c k\left(c_{11}^{0}+c_{22}^{0}\right) & =E\left(c_{12}^{\alpha}+c_{21}^{\alpha}\right), \\
0 & =E\left(c_{12}^{\alpha}-c_{21}^{\alpha}\right) .
\end{aligned}
$$

The last equation is uncoupled because it has different charge-conjugation parity $C$. One obtains the four orthonormal solutions, $\Psi_{i}$, for each $k, J$ given in the columns below

$$
\begin{array}{c|cccc|}
\multicolumn{1}{c}{} & \sqrt{2} \Psi_{++}^{0} & \sqrt{2} \Psi_{--}^{0} & \sqrt{2} \Psi_{S}^{0} & \sqrt{2} \Psi_{A}^{\alpha} \\
\cline { 2 - 5 }\left(c_{11}^{0}+c_{22}^{0}\right) & 1 & 1 & \cdot & \cdot \\
\left(c_{11}^{0}-c_{22}^{0}\right) & \frac{m c^{2}}{e} & -\frac{m c^{2}}{e} & \frac{\sqrt{2} \hbar c k}{e} & \cdot \\
\left(c_{12}^{\alpha}+c_{21}^{\alpha}\right) & -\frac{\hbar c k}{e} & \frac{\hbar c k}{e} & \frac{\sqrt{2} m c^{2}}{e} & \cdot \\
\left(c_{12}^{\alpha}-c_{21}^{\alpha}\right) & \cdot & \cdot & \cdot & \sqrt{2} \\
\cline { 2 - 5 } C & (-1)^{J} & (-1)^{J} & (-1)^{J} & (-1)^{J+1} \\
P & (-1)^{J+1} & (-1)^{J+1} & (-1)^{J+1} & (-1)^{J+1}
\end{array}
$$

The atomic states are labelled $\Psi_{++}^{\lambda}$ and $\Psi_{--}^{\lambda}$ with superscript $\lambda$ which corresponds to the dominant component $c_{i j}^{\lambda} \neq 0$ where $m c^{2} \gg \hbar c k$ and the anomalous states $\Psi_{S}^{\lambda}$ and $\Psi_{A}^{\lambda}$ are labelled with superscript $\lambda$ which corresponds to the dominant component $c_{i j}^{\lambda} \neq 0$ where $\hbar c k \gg m c^{2}$. Note that $\left(c_{11}^{0}+c_{22}^{0}\right)=0$ for the anomalous states $\Psi_{S}^{0}$ as in (31).

2. Case 2B: $S=1, L=J$

\section{Letting}

$$
\Psi=\frac{1}{\rho}\left(\begin{array}{c}
c_{11}^{1}|J, 1, k\rangle \\
c_{12}^{\beta}|J \beta, 1, k\rangle \\
c_{12}^{\beta}|J \beta, 1, k\rangle \\
c_{22}^{1}|J, 1, k\rangle
\end{array}\right),
$$

the two-body Dirac equation in the momentum basis, for a given $k, J$, gives the following three coupled equations for the symmetrized Bessel coefficients, 


$$
\begin{aligned}
2 m c^{2}\left(c_{11}^{1}+c_{22}^{1}\right)-2 \hbar c k\left(c_{12}^{\beta}-c_{21}^{\beta}\right) & =E\left(c_{11}^{1}-c_{22}^{1}\right), \\
2 m c^{2}\left(c_{11}^{1}-c_{22}^{1}\right) & =E\left(c_{11}^{1}+c_{22}^{1}\right), \\
-2 \hbar c k\left(c_{11}^{1}-c_{22}^{1}\right) & =E\left(c_{12}^{\beta}-c_{21}^{\beta}\right), \\
0 & =E\left(c_{12}^{\beta}+c_{21}^{\beta}\right) .
\end{aligned}
$$

The last equation is uncoupled because it has different charge-conjugation parity $C$. One obtains the four orthonormal solutions $\Psi_{i}$ for each $k, J$ in the columns below

$$
\begin{array}{c|cccc|} 
& \sqrt{2} \Psi_{++}^{1} & \sqrt{2} \Psi_{--}^{1} & \sqrt{2} \Psi_{S}^{1} & \sqrt{2} \Psi_{A}^{\beta} \\
\cline { 2 - 5 }\left(c_{11}^{1}-c_{22}^{1}\right) & 1 & 1 & \cdot & \cdot \\
\left(c_{11}^{1}+c_{22}^{1}\right) & \frac{m c^{2}}{e} & -\frac{m c^{2}}{e} & \frac{\sqrt{2} \hbar c k}{e} & \cdot \\
\left(c_{12}^{\beta}-c_{21}^{\beta}\right) & -\frac{\hbar c k}{e} & \frac{\hbar c k}{e} & \frac{\sqrt{2} m c^{2}}{e} & \cdot \\
\left(c_{12}^{\beta}+c_{21}^{\beta}\right) & \cdot & \cdot & \cdot & \sqrt{2} \\
C & (-1)^{J+1} & (-1)^{J+1} & (-1)^{J+1} & (-1)^{J} \\
P & (-1)^{J+1} & (-1)^{J+1} & (-1)^{J+1} & (-1)^{J+1}
\end{array} .
$$

3. Case 3B: $S=1, L \neq J$

Letting

$$
\Psi=\frac{1}{\rho}\left(\begin{array}{c}
c_{11}^{\alpha}|J \alpha, 1, k\rangle+c_{11}^{\beta}|J \beta, 1, k\rangle \\
c_{12}^{0}|J, 0, k\rangle+c_{12}^{1}|J, 1, k\rangle \\
c_{21}^{0}|J, 0, k\rangle+c_{21}^{1}|J, 1, k\rangle \\
c_{22}^{\alpha}|J \alpha, 1, k\rangle+c_{22}^{\beta}|J \beta, 1, k\rangle
\end{array}\right),
$$

the two-body Dirac equation in the momentum basis, for a given $k, J$, gives the following coupled equations for the symmetrized Bessel coefficients, 


$$
\begin{aligned}
2 m c^{2}\left(c_{11}^{\alpha}-c_{22}^{\alpha}\right)-2 \hbar c k\left(c_{12}^{0}+c_{21}^{0}\right) & =E\left(c_{11}^{\alpha}+c_{22}^{\alpha}\right), \\
2 m c^{2}\left(c_{11}^{\alpha}+c_{22}^{\alpha}\right) & =E\left(c_{11}^{\alpha}-c_{22}^{\alpha}\right), \\
-2 \hbar c k\left(c_{11}^{\alpha}+c_{22}^{\alpha}\right) & =E\left(c_{12}^{0}+c_{21}^{0}\right), \\
2 m c^{2}\left(c_{11}^{\beta}+c_{22}^{\beta}\right)-2 \hbar c k\left(c_{12}^{1}-c_{21}^{1}\right) & =E\left(c_{11}^{\beta}-c_{22}^{\beta}\right), \\
2 m c^{2}\left(c_{11}^{\beta}-c_{22}^{\beta}\right) & =E\left(c_{11}^{\beta}+c_{22}^{\beta}\right), \\
-2 \hbar c k\left(c_{11}^{\beta}-c_{22}^{\beta}\right) & =E\left(c_{12}^{1}-c_{21}^{1}\right), \\
0 & =E\left(c_{12}^{0}-c_{21}^{0}\right), \\
0 & =E\left(c_{12}^{1}+c_{21}^{1}\right) .
\end{aligned}
$$

The first six equations consist of two sets of three coupled equations. The last two equations are uncoupled because they have different charge-conjugation parity $C$. As in the coordinate representation, these equations can be found from Case 1B and Case 2B by the exchange $m_{e} \longleftrightarrow-m_{e}, c_{11} \longleftrightarrow c_{21}, c_{22} \longleftrightarrow c_{12}$, or, equivalently, the exchange $m_{p} \longleftrightarrow-m_{p}$, $c_{11} \longleftrightarrow c_{12}, c_{22} \longleftrightarrow c_{21}$. One obtains the eight orthonormal solutions $\Psi_{i}$ for each $k, J$ in the columns below

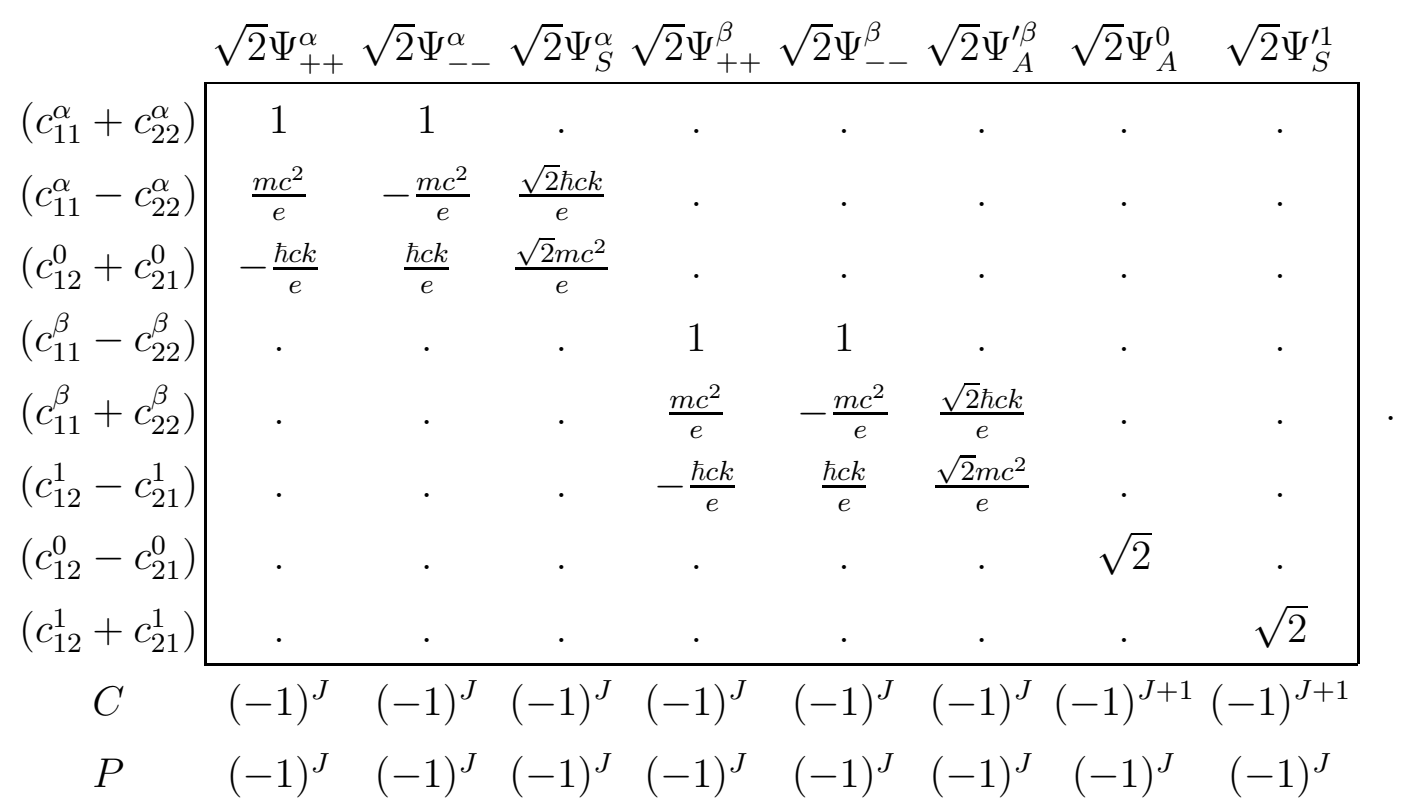

Here the prime superscript is used for the anomalous wave functions $\Psi_{A}^{\prime \beta}$ and $\Psi_{S}^{\prime 1}$ to distinguish them from their Case $2 \mathrm{~B}$ counterparts which have the same $C$ but different $P$. As seen in (B9), the two sets of three equations are separable for free particles just like Case 
1B and Case 2B, but will be coupled by the Coulomb potential because they have the same $C$ and $P$.

\section{Addition Theorems.}

Four important addition theorems can be derived for the products of single particle functions of free particles $g_{n_{e}}^{\ell_{e}} j_{e}\left(k r_{e}, \theta_{e}, \varphi_{e}\right) g_{n_{p}}^{\ell_{p} j_{p}}\left(k r_{p}, \theta_{p}, \varphi_{p}\right)$ with coordinates $\boldsymbol{r}_{e}, \boldsymbol{r}_{p}$ where

$$
\begin{aligned}
g_{n}^{\ell j}(k r, \theta, \varphi) & \equiv j_{\ell}(k r)\left[Y^{\ell}(\theta, \varphi) \chi^{\frac{1}{2}}\right]_{n}^{j}, \\
& =j_{\ell}(k r) \sum_{m, \sigma} C_{m \sigma n}^{\ell \frac{1}{2} j} Y_{m}^{\ell} \chi_{\sigma}^{\frac{1}{2}},
\end{aligned}
$$

and

$$
\left[\begin{array}{llll}
g_{n_{e}}^{j_{e} \pm \frac{1}{2}} & j_{e} & j_{p} \pm \frac{1}{2} & j_{p} \\
g_{n_{p}}
\end{array}\right]_{N}^{J}=\sum_{n_{e}, n_{p}} C_{n_{e} n_{p} N}^{j_{e}} j_{p} J g_{n_{e}}^{j_{e} \pm \frac{1}{2}} j^{j_{e}} g_{n_{p}}^{j_{p} \pm \frac{1}{2}} j_{p}
$$

The four possible states, $j_{L}(k \rho)\left[Y^{L}\left(\theta_{\rho}, \varphi_{\rho}\right) \Omega^{S}\right]_{N}^{J}$, for a given $J$ with relative coordinates $\boldsymbol{\rho}=\boldsymbol{r}_{e}-\boldsymbol{r}_{p}$ can be expanded in terms of these products (B11). These four addition theorems are derived here from the work of Danos and Maximon [20].

Using the recoupling coefficients $a$ and $b$ in (16) and ignoring normalizations $N_{J k}$ in (20) and (21), one obtains

$$
\begin{aligned}
& |J, 0, k\rangle / \rho=j_{J}(k \rho)\left[Y^{J}\left(\theta_{\rho}, \varphi_{\rho}\right) \Omega^{0}\right]_{N}^{J}
\end{aligned}
$$

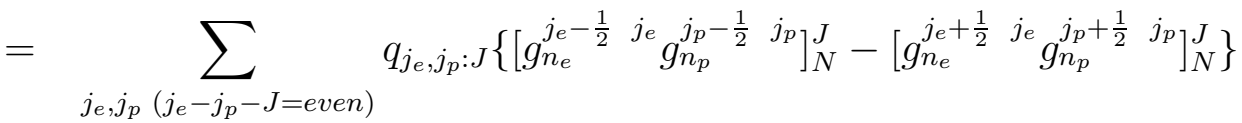

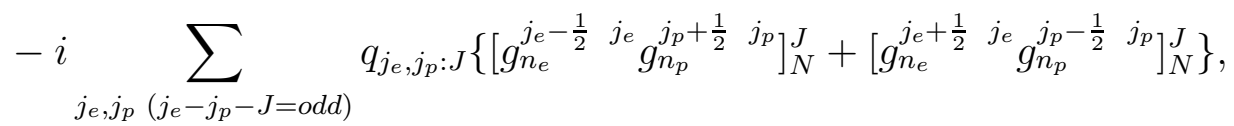

and

$$
\begin{aligned}
& |J \alpha, 1, k\rangle / \rho=i a j_{J+1}(k \rho)\left[Y^{J+1}\left(\theta_{\rho}, \varphi_{\rho}\right) \Omega^{1}\right]_{N}^{J}+i b j_{J-1}(k \rho)\left[Y^{J-1}\left(\theta_{\rho}, \varphi_{\rho}\right) \Omega^{1}\right]_{N}^{J}
\end{aligned}
$$

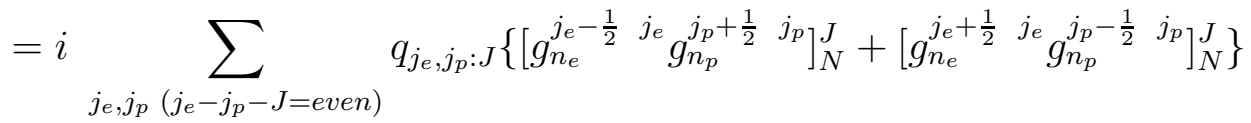

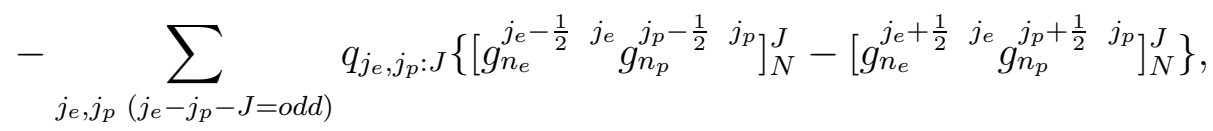

where 


$$
q_{j_{e}, j_{p}: J}=i^{\left(j_{e}-j_{p}-J\right)}\left(\frac{2 \pi\left[j_{e}\right]\left[j_{p}\right]}{[J]}\right)^{\frac{1}{2}} C_{\frac{1}{2}-\frac{1}{2} 0}^{j_{e}} j_{p} J
$$

One also obtains

$$
\begin{aligned}
|J, 1, k\rangle / \rho=j_{J}(k \rho)\left[Y^{J}\left(\theta_{\rho}, \varphi_{\rho}\right) \Omega^{1}\right]_{N}^{J} & \\
=- & \sum_{j_{e}, j_{p}\left(j_{e}-j_{p}-J=e v e n\right)} p_{j_{e}, j_{p}: J}\left\{\left[g_{n_{e}}^{j_{e}-\frac{1}{2}} j^{j_{e}} g_{n_{p}}^{j_{p}-\frac{1}{2}} j^{j_{p}}\right]_{N}^{J}+\left[g_{n_{e}}^{j_{e}+\frac{1}{2}} j_{e}^{j_{e}} g_{n_{p}}^{j_{p}+\frac{1}{2}}{ }^{j_{p}}\right]_{N}^{J}\right\} \\
& \quad-i \sum_{j_{e}, j_{p}\left(j_{e}-j_{p}-J=o d d\right)} p_{j_{e}, j_{p}: J}\left\{\left[g_{n_{e}}^{j_{e}-\frac{1}{2}} j_{e} g_{n_{p}}^{j_{p}+\frac{1}{2}} j_{p}^{j_{p}}\right]_{N}^{J}-\left[g_{n_{e}}^{j_{e}+\frac{1}{2}} j_{e} g_{n_{p}}^{j_{p}-\frac{1}{2}} j^{j_{p}}\right]_{N}^{J}\right\},
\end{aligned}
$$

and

$$
\begin{aligned}
& |J \beta, 1, k\rangle / \rho=-i b j_{J+1}(k \rho)\left[Y^{J+1}\left(\theta_{\rho}, \varphi_{\rho}\right) \Omega^{1}\right]_{N}^{J}+i a j_{J-1}(k \rho)\left[Y^{J-1}\left(\theta_{\rho}, \varphi_{\rho}\right) \Omega^{1}\right]_{N}^{J}
\end{aligned}
$$

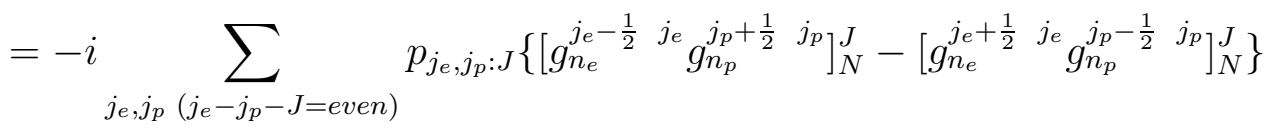

$$
\begin{aligned}
& -\sum_{j_{e}, j_{p}\left(j_{e}-j_{p}-J=o d d\right)} p_{j_{e}, j_{p}: J}\left\{\left[\begin{array}{llll}
g_{n_{e}}^{j_{e}-\frac{1}{2}} & j_{e} & j_{n_{p}}-\frac{1}{2} & j_{p}
\end{array}\right]_{N}^{J}+\left[\begin{array}{lll}
g_{n_{e}}^{j_{e}+\frac{1}{2}} & j_{e} & g_{n_{p}+\frac{1}{2}}^{j_{p}} j_{p}
\end{array}\right]_{N}^{J}\right\}
\end{aligned}
$$

where

$$
p_{j_{e}, j_{p}: J}=i^{\left(j_{e}-j_{p}-J\right)}\left(\frac{2 \pi\left[j_{e}\right]\left[j_{p}\right]}{[J]}\right)^{\frac{1}{2}} C_{\frac{1}{2} \frac{1}{2} 1}^{j_{e} j_{p} J} .
$$

One can readily evaluate the expressions $(\overline{\mathrm{B} 12})$ and $(\overline{\mathrm{B} 15})$ for the special case of $J=0$ so that

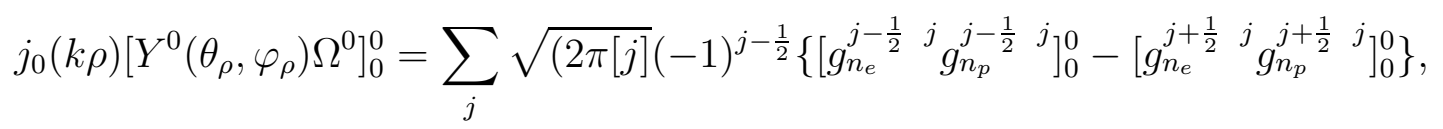

and

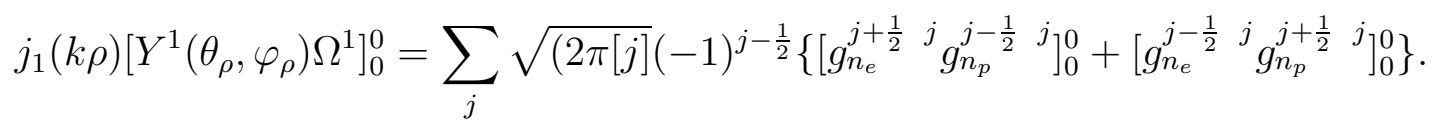

These two special cases have been given previously [21].

\section{Acknowledgments}

The author would like to give special thanks to Drs. Tony Scott, Janine Shertzer, Gordon Drake, and William Harter. The author has benefitted greatly from the work of Scott, 
Shertzer, and Moore [1] and considers this work, in many respects, to be an extension of theirs. Drs. Scott and Shertzer were very helpful in explaining their finite element calculations which were duplicated here in order to show the anomalous wave functions. Particular credit goes to Dr. Drake for suggesting the separability between the anomalous and atomic states. Dr. Harter has been encouraging this work for a number of years. Thanks are also due to Dr. Joel Kress for extending the hospitality of Theoretical Division, Los Alamos National Laboratory and to Drs. Arthur Voter, Brian Kendrick, Peter Miloni, and Lee Collins for many helpful suggestions during my time at Los Alamos. Dr. Kendrick suggested the pertinence of the discrete variable representation for the anomalous states. Finally, the author would like to thank Drs. Robert Sang and Max Standage for their help and support while at Griffith University.

\section{References}

[1] T. C. Scott, J. Shertzer, and R. A. Moore, Phys. Rev. A 45, 4393 (1992).

[2] H. A. Bethe and E. E. Salpeter, Quantum Mechanics of One- and Two-Electron Atoms (Academic Press, New York, 1957).

[3] E. E. Salpeter and H. A. Bethe, Phys. Rev. 84, 1232 (1951).

[4] J. Malenfant, Phys. Rev. D 38, 3295 (1988).

[5] J. J. Sakurai, Advanced Quantum Mechanics (Addison-Wesley, New York, 1967).

[6] R. A. Ferrell, Phys. Rev. 84, 858 (1951).

[7] T. Ishidzu, Progr. Theoret. Phys. 6, 48 (1951).

[8] T. Ishidzu, Progr. Theoret. Phys. 6, 154 (1951).

[9] T. Fulton and P. C. Martin, Phys. Rev. 95, 811 (1954).

[10] J. C. Light and T. Carrington, Adv. Chem. Phys. 114, 263 (2000).

[11] P. V. Alstine and H. W. Crater, Found. Phys. 27, 67 (1997).

[12] M. Gell-Mann and F. Low, Phys. Rev. 84, 350 (1951).

[13] J. Sucher, Phys. Rev. 107, 1448 (1957).

[14] J. Sapirstein and D. Yennie, Adv.Ser.Direct.High Energy Phys. 7, 560 (1990). 
[15] H. Ito, arXiv: hep-ph/9708268v1 (1997).

[16] I. P. Grant, Relativistic Quantum Theory of Atomcs and Molecules: Theory and Computation (Springer, 2007).

[17] R. P. Feynman, Phys. Rev. 76, 749 (1949).

[18] E. E. Salpeter, Phys. Rev. 87, 328 (1952).

[19] R. Karplus and A. Klein, Phys. Rev. 87, 848 (1952).

[20] M. Danos and L. C. Maximon, J. Math. Phys. 6, 766 (1965).

[21] M. Abramowitz and I. A. Stegun, Handbook of Mathematical Functions (Dover Publishing, New York, 1972). 


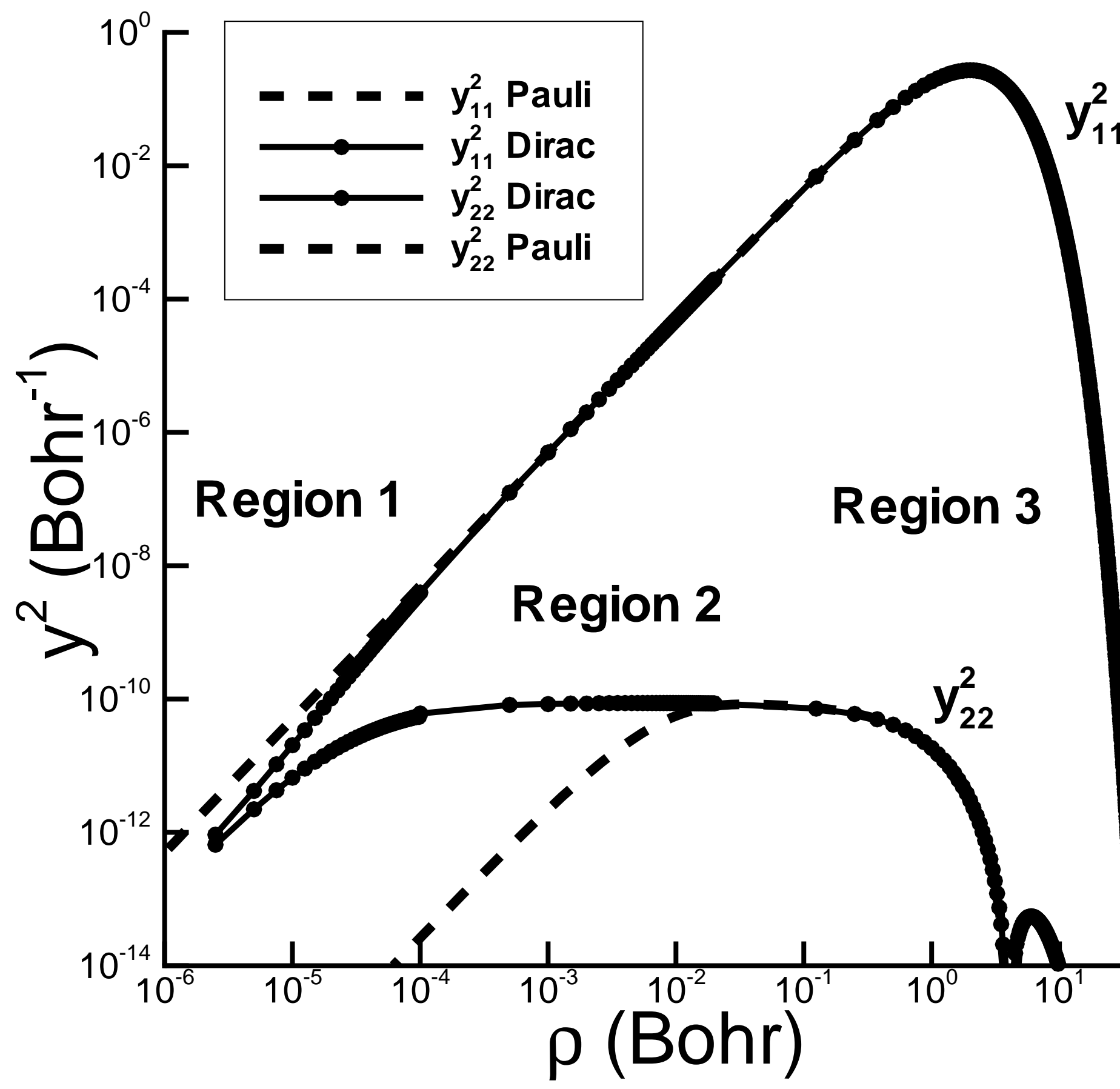




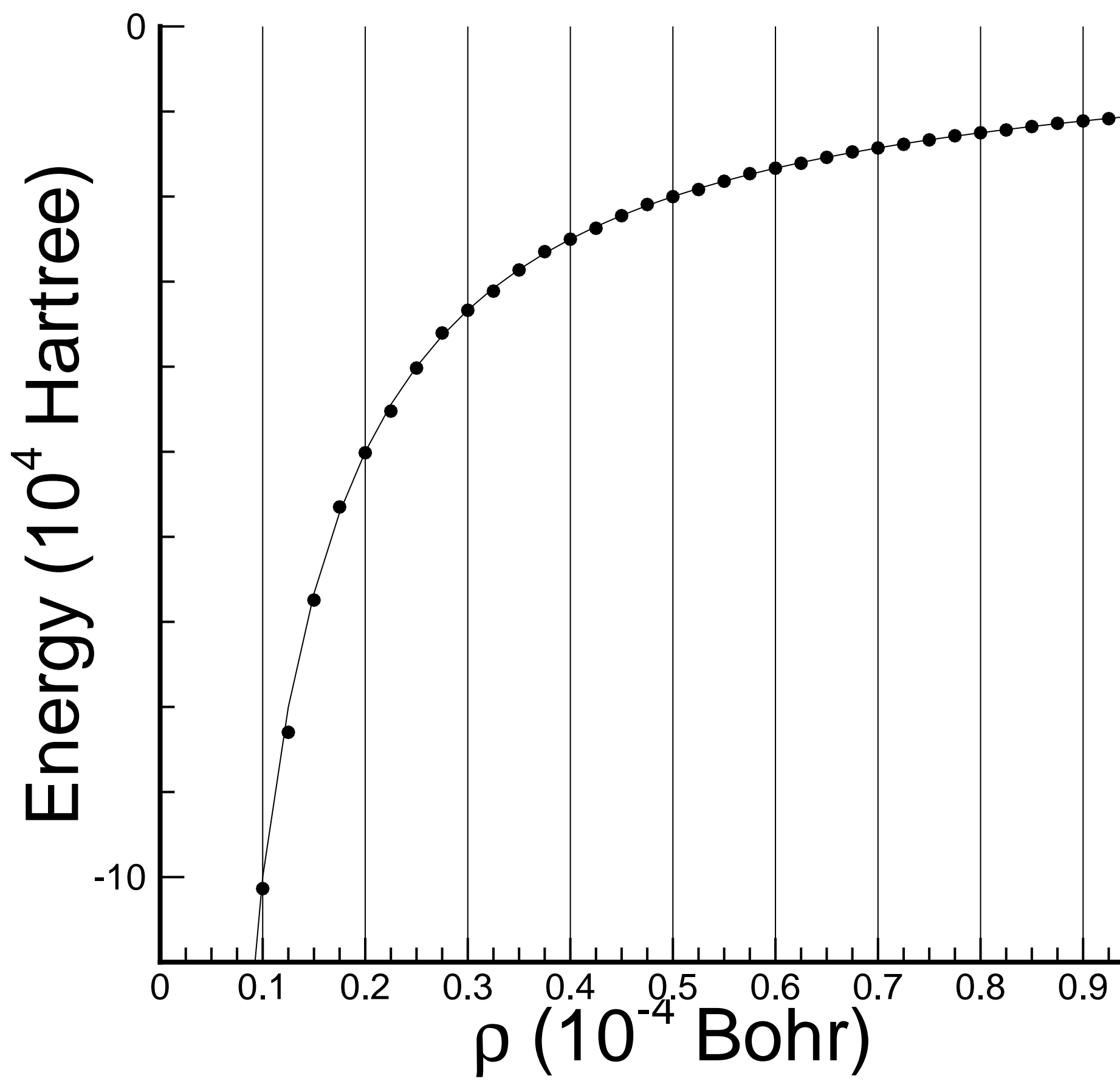




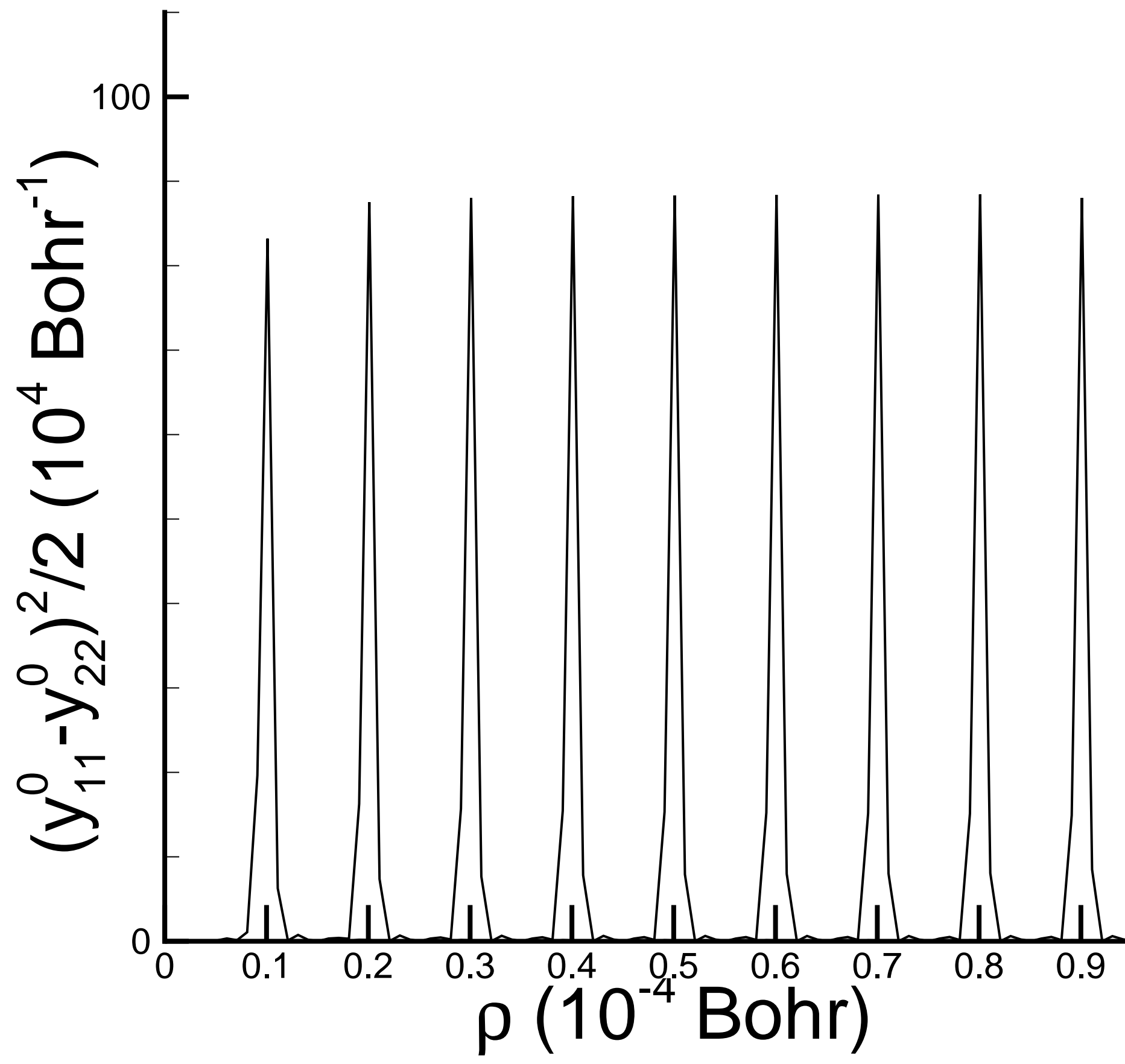




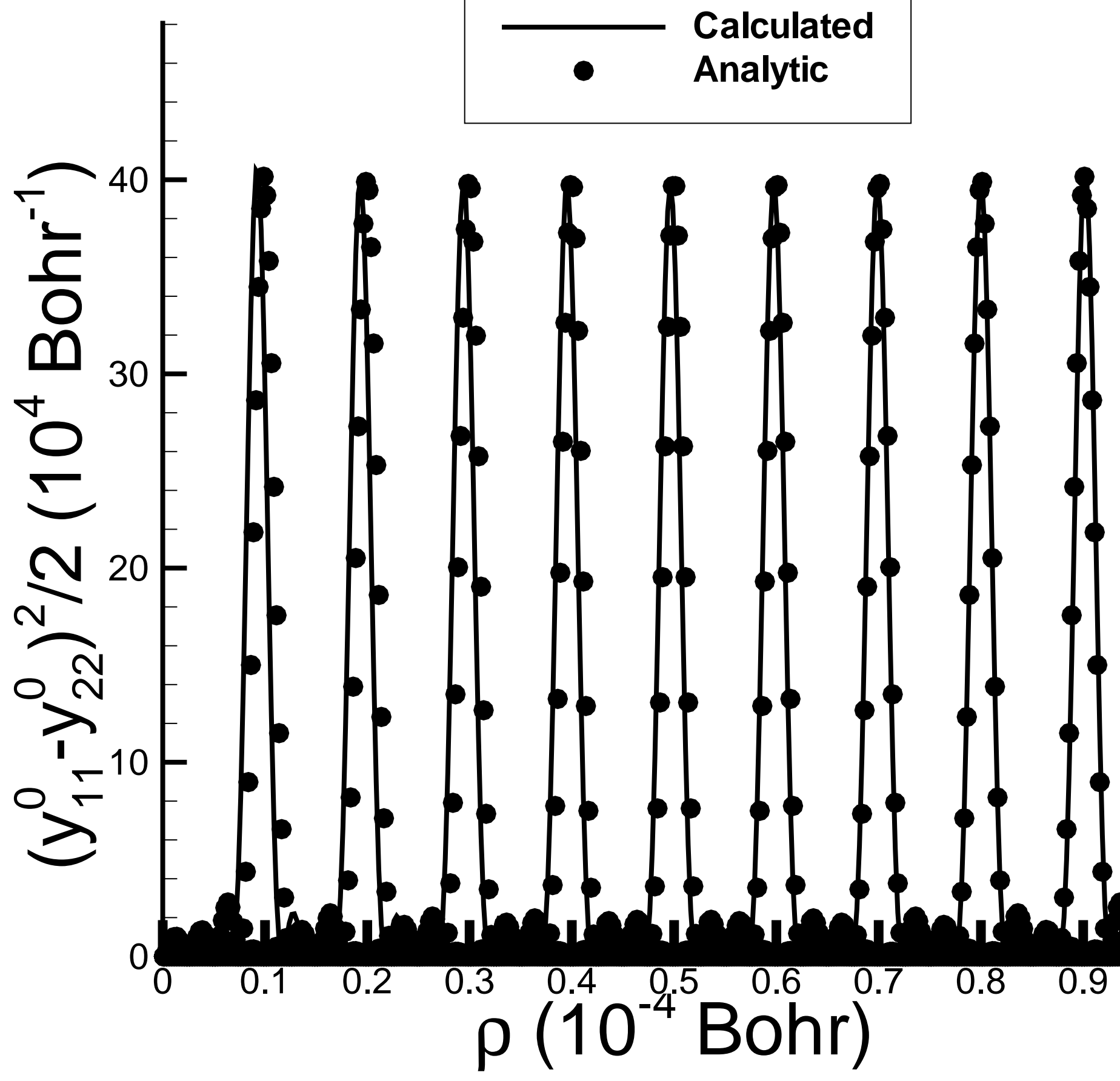




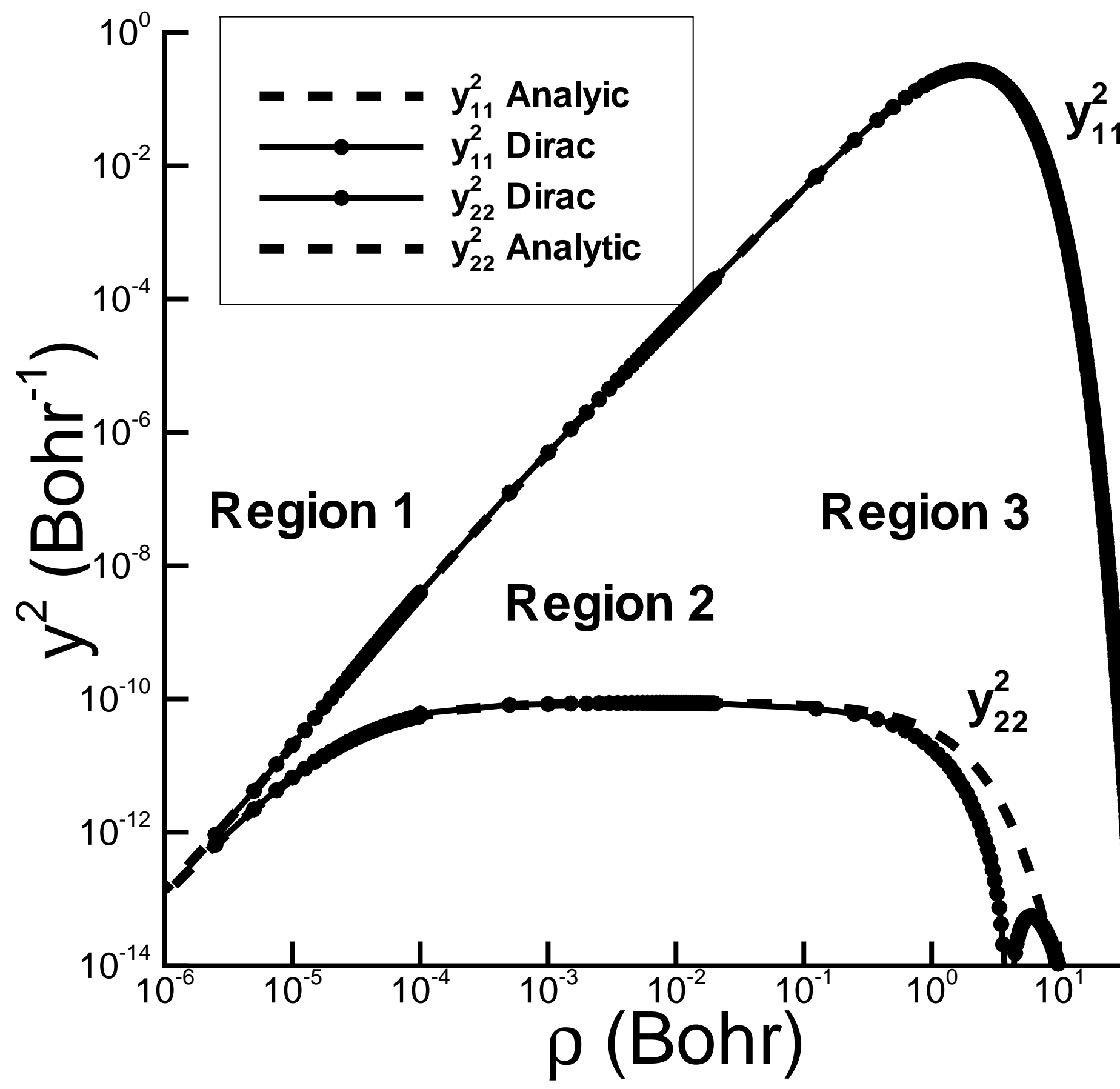




\section{FIGURE CAPTIONS}

FIG. 1. The Dirac and Pauli radial wavefunction components (squared) for the groundstate are compared. Large dots correspond to the 399 grid points used for the finite element calculation. Plots are on a log-log scale.

FIG. 2. The energies $E_{i}$ for the anomalous states are found by solving the same equations as in Fig. 1. The finite element grid points $\rho_{i}$ shown here are the same as in Region 1 of Fig. 1. Energies are given analytically by $-1 / \rho_{i}$ Hartree shown by the solid line. Verticle lines are at the element boundaries.

FIG. 3. The anomalous bound state wavefunctions in the coordinate representation for the energies in Fig. 2 at the element boundaries. The wavefunctions are approximately evenly spaced delta functions. The component $\left(y_{12}^{+}+y_{21}^{+}\right)$is too small to be seen.

FIG. 4. Anomalous bound-state wavefunctions as in Fig. 3 but calculated in the momentum representation. The component $\left(y_{12}^{\alpha}+y_{21}^{\alpha}\right)$ is too small to be seen. Analytic results are from (38).

FIG. 5. The Dirac radial wavefunctions in Fig. 1 are compared with analytical results in which the Dirac zero-order ground-state $\left|\Psi_{D}\right\rangle^{(0)}$ is coupled to the anomalous states $\left|\Psi_{S}^{0}, i\right\rangle$ by the Coulomb potential. Plots are on a log-log scale. 